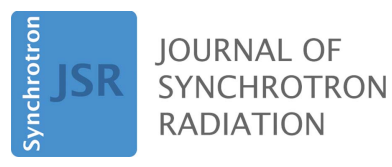

ISSN 1600-5775

Received 25 May 2017

Accepted 14 August 2017

Edited by M. Yabashi, RIKEN SPring-8 Center, Japan

Keywords: X-ray; lens; oval; nanofocusing; aberration.

\section{Aberration-free aspherical lens shape for shortening the focal distance of an already convergent beam}

\author{
John P. Sutter* and Lucia Alianelli \\ Diamond Light Source Ltd, Chilton, Didcot, Oxfordshire OX11 ODE, UK. \\ *Correspondence e-mail: john.sutter@diamond.ac.uk
}

The shapes of single lens surfaces capable of focusing divergent and collimated beams without aberration have already been calculated. However, nanofocusing compound refractive lenses (CRLs) require many consecutive lens surfaces. Here a theoretical example of an X-ray nanofocusing CRL with 48 consecutive surfaces is studied. The surfaces on the downstream end of this CRL accept $\mathrm{X}$-rays that are already converging toward a focus, and refract them toward a new focal point that is closer to the surface. This case, so far missing from the literature, is treated here. The ideal surface for aberration-free focusing of a convergent incident beam is found by analytical computation and by ray tracing to be one sheet of a Cartesian oval. An 'X-ray approximation' of the Cartesian oval is worked out for the case of small change in index of refraction across the lens surface. The paraxial approximation of this surface is described. These results will assist the development of large-aperture CRLs for nanofocusing.

\section{Introduction}

Compound refractive lenses (CRLs) have been used to focus X-ray beams since Snigirev et al. (1996) demonstrated that the extremely weak refraction of X-rays by a single lens surface could be reinforced by lining up a series of lenses. As the X-ray focal spot size has been brought down below $1 \mu \mathrm{m}$, more lenses have been necessary to achieve the very short focal lengths required. Because the absorption of X-rays in the lens material is generally significant, it thus becomes critical to design the CRL with the shortest length possible for the given focal length in order to minimize the thickness of the refractive material through which the X-rays must pass.

In recent years, designs for novel nanofocusing lenses have been proposed. X-ray refractive lenses will deliver ideally focused beam of nanometer size if the following conditions can be satisfied:

(1) The lens material should not introduce unwanted scattering.

(2) The fabrication process should not introduce shape errors or roughness above a certain threshold.

(3) Absorption should be minimized in order to increase the lens effective aperture.

(4) The lens designs should not introduce geometrical aberrations.

In response to the first three of these conditions, planar micro-fabrication methods including electron beam lithography, silicon etch and LIGA have successfully been used to fabricate planar parabolic CRLs, single-element parabolic kinoform lenses (Aristov et al., 2000) and single-element elliptical kinoform lenses (Evans-Lutterodt et al., 2003) from 
silicon. However, the best focus that can be obtained is strongly dependent on material and X-ray energy. A collimating-focusing pair of elliptical silicon kinoform lenses with a focal length of $75 \mathrm{~mm}$ has successfully focused $8 \mathrm{keV}$ photons from an undulator source of $45 \pm 5 \mu \mathrm{m}$ full width at half-maximum (FWHM) into a spot of $225 \mathrm{~nm}$ FWHM (Alianelli et al., 2011). On the other hand, although silicon lenses can be fabricated with very high accuracy, they are too absorbing to deliver a focused beam below $100 \mathrm{~nm}$ at energy values below $12 \mathrm{keV}$, unless kinoform lenses with extremely small sidewalls can be manufactured. As a result, diamond has come to be viewed as a useful material for CRLs. Diamond, along with beryllium and boron, is one of the ideal candidates to make X-ray lenses due to good refractive power, low absorption and excellent thermal properties. Planar refractive lenses made from diamond were demonstrated by Nöhammer et al. (2003). Fox et al. (2014) have focused an X-ray beam of $15 \mathrm{keV}$ to a $230 \mathrm{~nm}$ spot using a microcrystalline diamond lens, and an X-ray beam of $11 \mathrm{keV}$ to a $210 \mathrm{~nm}$ spot using a nanocrystalline diamond lens. Designs of diamond CRLs proposed by Alianelli et al. (2016) would potentially be capable of focusing down to $50 \mathrm{~nm}$ beam sizes. Many technical problems remain to be solved in the machining of diamond; however, our current aim is to provide an ideal lens design to be used when the technological issues are overcome. We assume that technology in both reductive and additive techniques will advance in the coming decade and that X-ray refractive lenses with details of several tens of nanometers will be fabricated. This will make X-ray refractive optics more competitive than they are today for the ultra-short focal lengths. When that happens, lens designs that do not introduce aberrations will be crucial.

The aim of this paper is to define a nanofocusing CRL with the largest possible aperture that can be achieved without introducing aberrations to the focus. The determination of the ideal shape of each lens surface becomes more critical as the desired aperture grows. Suzuki (2004) states that the ideal lens surface for focusing a plane wave is an ellipsoid, although in fact this is true only if the index of refraction increases as the X-rays cross the surface [see Sanchez del Rio \& Alianelli (2012) and references therein, as well as $\$ 2.4$ of this paper]. The same author also proposes the use of two ellipsoidal lenses for point-to-point focusing, but a nanofocusing X-ray
CRL requires a much larger number of lenses because of the small refractive power and the short focal length. EvansLutterodt et al. (2007), in their demonstration of the ability of kinoform lenses to exceed the numerical aperture set by the critical angle, used Fermat's theorem to calculate the ideal shapes of their four lenses, but explicitly described the shape of only the first lens (an ellipse). Sanchez del Rio \& Alianelli (2012) pointed out the general answer, known for centuries, that the ideal shape of a lens surface for focusing a point source to a point image is not a conic section (a curve described by a second-degree polynomial). Rather, it is a Cartesian oval, which is a type of quartic curve (i.e. a curve described by a fourth-degree, or quartic, polynomial). An array of sections of Cartesian ovals is therefore one possible solution to the task of designing an X-ray lens for single-digit nanometer focusing. Previous authors in X-ray optics have not calculated analytical solutions (which do exist), but instead relied on numerical calculations of the roots without asking how well conditioned the quartic polynomial is; that is, how stable the roots of the polynomial are against small changes in its coefficients. However, in this paper it will be shown that finite numerical precision can cause errors in the calculation of the Cartesian oval when the change in refractive index across the lens surface becomes very small, as is usually the case with $\mathrm{X}$-rays. Moreover, it has not been made explicit in the literature when it is reasonable to approximate the ideal Cartesian oval with various conic sections (ellipses, hyperbolas or parabolas). As a result, for the sake of rigor, the authors have considered it worthwhile to find the analytical solutions explicitly. This has not been done before in any recent papers. Finally, Alianelli et al. (2015) state that no analytical solution exists for a lens surface that accepts an incident beam converging to a point and that focuses this beam to another point closer to the lens surface. This paper will concentrate on that very case and will show that in fact such a lens surface can be described by a Cartesian oval. The existence of such solutions removes the necessity of using pairs of lens surfaces of which the first slightly focuses the beam and the second collimates the beam again.

Schroer \& Lengeler (2005) proposed the construction of 'adiabatically' focusing CRLs, in which the aperture of each lens follows the width of the X-ray beam as the beam converges to its focus. Fig. 1 displays a schematic of an adia-

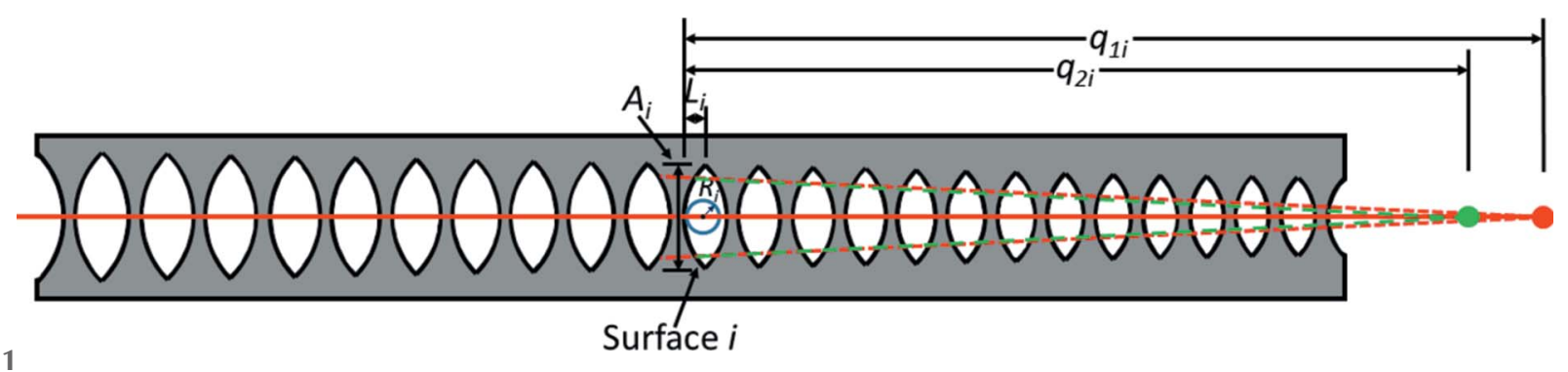

Figure 1

Surface $i$

Schematic drawing of an adiabatically focusing CRL for X-rays. The X-ray beam runs along the central axis from left to right. $A_{i}, R_{i}$ and $L_{i}$ are, respectively, the geometrical aperture, the radius at the apex and the length along the beam direction of the $i$ th lens surface. If the lens surfaces are assumed to be parabolic as in Schroer \& Lengeler (2005), $L_{i}=A_{i}^{2} /\left(8 R_{i}\right) . q_{1 i}$ and $q_{2 i}$ are, respectively, the distance of the object and the distance of the image of the $i$ th lens surface from that surface's apex. 
Table 1

Lens surfaces proposed for a diamond nanofocusing CRL for X-rays of energy $15 \mathrm{keV}$.

The distance from the apex of the last lens surface to the final focal plane is $11.017 \mathrm{~mm} . i$ is the place of each surface in the CRL. $R_{i}$ is the radius at the apex. $f_{i}$ is the focal length. $A_{i}$ is the geometrical aperture. $q_{1 i}$ is the downstream distance of the object of lens surface $i$. (A negative value of $q_{1 i}$ means that the object of lens surface $i$ is upstream.) $q_{2 i}$ is the downstream distance of the image of lens surface $i$. The distance between the apices of consecutive lens surfaces is $0.005 \mathrm{~mm}$. For legibility, $R_{i}$ and $A_{i}$ are rounded to four significant digits, and $f_{i}, q_{1 i}$ and $q_{2 i}$ are rounded to three decimal places.

\begin{tabular}{|c|c|c|c|c|c|}
\hline$i$ & $R_{i}(\mathrm{~mm})$ & $f_{i}(\mathrm{~mm})$ & $A_{i}(\mathrm{~mm})$ & $q_{1 i}(\mathrm{~mm})$ & $q_{2 i}(\mathrm{~mm})$ \\
\hline 1 & 0.05 & 15484.670 & 0.075 & -46999.864 & 23092.905 \\
\hline 2 & 0.045 & 13936.203 & 0.07425 & 23092.900 & 8691.196 \\
\hline 3 & 0.0405 & 12542.583 & 0.07351 & 8691.191 & 5133.801 \\
\hline 4 & 0.03645 & 11288.325 & 0.07277 & 5133.796 & 3528.896 \\
\hline 5 & 0.03281 & 10159.492 & 0.07204 & 3528.891 & 2619.136 \\
\hline 6 & 0.02952 & 9143.543 & 0.07132 & 2619.131 & 2035.944 \\
\hline 7 & 0.02657 & 8229.189 & 0.07061 & 2035.939 & 1632.140 \\
\hline 8 & 0.02391 & 7406.270 & 0.06990 & 1632.135 & 1337.408 \\
\hline 9 & 0.02152 & 6665.643 & 0.06921 & 1337.403 & 1113.907 \\
\hline 10 & 0.01937 & 5999.078 & 0.06851 & 1113.902 & 939.463 \\
\hline 11 & 0.01743 & 5399.171 & 0.06783 & 939.458 & 800.220 \\
\hline 12 & 0.01569 & 4859.254 & 0.06715 & 800.215 & 687.069 \\
\hline 13 & 0.01412 & 4373.328 & 0.06648 & 687.064 & 593.780 \\
\hline 14 & 0.01271 & 3935.995 & 0.06581 & 593.775 & 515.941 \\
\hline 15 & 0.01144 & 3542.396 & 0.06516 & 515.936 & 450.345 \\
\hline 16 & 0.01029 & 3188.156 & 0.06450 & 450.340 & 394.601 \\
\hline 17 & 0.009265 & 2869.341 & 0.06386 & 394.596 & 346.891 \\
\hline 18 & 0.008339 & 2582.407 & 0.06322 & 346.886 & 305.808 \\
\hline 19 & 0.007505 & 2324.166 & 0.06259 & 305.803 & 270.245 \\
\hline 20 & 0.006754 & 2091.749 & 0.06196 & 270.240 & 239.322 \\
\hline 21 & 0.006079 & 1882.574 & 0.06134 & 239.317 & 212.325 \\
\hline 22 & 0.005471 & 1694.317 & 0.06073 & 212.320 & 188.677 \\
\hline 23 & 0.004924 & 1524.885 & 0.06012 & 188.672 & 167.898 \\
\hline 24 & 0.004432 & 1372.397 & 0.05952 & 167.893 & 149.592 \\
\hline 25 & 0.003988 & 1235.157 & 0.05893 & 149.587 & 133.428 \\
\hline 26 & 0.003590 & 1111.641 & 0.05834 & 133.423 & 119.125 \\
\hline 27 & 0.003231 & 1000.477 & 0.05775 & 119.120 & 106.446 \\
\hline 28 & 0.002908 & 900.429 & 0.05718 & 106.441 & 95.189 \\
\hline 29 & 0.002617 & 810.387 & 0.05660 & 95.184 & 85.179 \\
\hline 30 & 0.002355 & 729.348 & 0.05604 & 85.174 & 76.268 \\
\hline 31 & 0.002120 & 656.413 & 0.05548 & 76.263 & 68.325 \\
\hline 32 & 0.001908 & 590.772 & 0.05492 & 68.320 & 61.238 \\
\hline 33 & 0.001717 & 531.695 & 0.05437 & 61.233 & 54.909 \\
\hline 34 & 0.001545 & 478.525 & 0.05383 & 54.904 & 49.253 \\
\hline 35 & 0.001391 & 430.673 & 0.05329 & 49.248 & 44.194 \\
\hline 36 & 0.001252 & 387.605 & 0.05276 & 44.189 & 39.667 \\
\hline 37 & 0.001126 & 348.845 & 0.05223 & 39.662 & 35.613 \\
\hline 38 & 0.001014 & 313.960 & 0.05171 & 35.608 & 31.981 \\
\hline 39 & 0.0009124 & 282.564 & 0.05119 & 31.976 & 28.725 \\
\hline 40 & 0.0008212 & 254.308 & 0.05068 & 28.720 & 25.806 \\
\hline 41 & 0.0007390 & 228.877 & 0.05017 & 25.801 & 23.187 \\
\hline 42 & 0.0006651 & 205.989 & 0.04967 & 23.182 & 20.837 \\
\hline 43 & 0.0005986 & 185.390 & 0.04917 & 20.832 & 18.728 \\
\hline 44 & 0.0005388 & 166.851 & 0.04868 & 18.723 & 16.834 \\
\hline 45 & 0.0004849 & 150.166 & 0.04820 & 16.829 & 15.133 \\
\hline 46 & 0.0004364 & 135.150 & 0.04771 & 15.128 & 13.605 \\
\hline 47 & 0.0003928 & 121.635 & 0.04724 & 13.600 & 12.232 \\
\hline 48 & 0.0003535 & 109.471 & 0.04676 & 12.227 & 10.999 \\
\hline
\end{tabular}

batic CRL. A potential example of such a CRL, which would be made from diamond, is given in Table 1. This example treats X-rays of energy $15 \mathrm{keV}$, for which diamond has an index of refraction $1-\Delta$ where $\Delta=3.23 \times 10^{-6}$. The very small difference between the index of refraction of diamond and that of vacuum is typical for X-ray lenses. Calculations of surfaces 2, 24 and 48 of Table 1 will be demonstrated in the following treatment. The loss of numerical precision when using the exact analytical solutions of the Cartesian oval at small $\Delta$ will be avoided by an approximation of the quartic Cartesian oval equation to lowest order in $\Delta$. The cubic equation resulting from this 'X-ray approximation' will be shown to be numerically stable. The approximate cubic and the exact quartic equation will be shown to agree when the latter is numerically tractable. As in Sanchez del Rio \& Alianelli (2012), conic approximations will be made to the ideal surface in the paraxial case. The results of this paper agree with theirs in showing that Cartesian ovals, even when calculated by the cubic 'X-ray' approximation, introduce no detectable aberrations, and that elliptical or hyperbolic lens surfaces introduce less aberration than the usually used parabolic surfaces. However, this paper will also demonstrate that at sufficiently high apertures even the elliptical or hyperbolic approximation will produce visible tails in the focal spot. This is especially true for surface 48 , the final surface and the one with the smallest focal length, where the elliptical and hyperbolic approximation produces tails in the focus at an aperture only slightly larger than that given in Table 1.

At the end of this treatment, the focal spot profiles calculated by ray tracing for the compound refractive lens (CRL) of Table 1 will be compared with the diffraction broadening that inevitably results from the limited aperture. The absorption in the lens material limits the passage of X-rays through a CRL to an effective aperture $A_{\text {eff }}$ that is smaller than the geometrical aperture. This in turn restricts the numerical aperture (NA) of a CRL of $N$ surfaces to a value $A_{\text {eff }} /\left(2 q_{2 N}\right)$, where $q_{2 N}$ is the distance from the last ( $N$ th) surface to the final focus. Lengeler et al. (1999) derive a FWHM of $0.75 \lambda /(2 \mathrm{NA})$ for the Airy disk at the focal spot. This yields the diffraction broadening and hence the spatial resolving power of the CRL. Formulas for the effective aperture of a CRL in which all lens surfaces are identical have been derived by Lengeler et al. (1998, 1999), and Schroer \& Lengeler (2005) have derived formulas for the effective aperture of an adiabatically focusing CRL. Very recently Kohn (2017) has re-examined the calculation of the effective aperture, surveying the various definitions appearing in the literature and distinguishing carefully between one-dimensionally and two-dimensionally focusing CRLs. In this paper the effective aperture and the numerical aperture will be estimated numerically by ray tracing, taking full account of the absorption to which each ray is subjected along the path from the source to the focus. For simplicity, the lens surfaces will all be assumed to be one-dimensionally focusing parabolic cylinders. It will be shown that the parabola is an adequate approximation to the ideal Cartesian oval within the effective aperture of the CRL in Table 1.

\section{Principles}

\subsection{Definitions and derivation of ideal lens surface}

The first task of this article is to calculate the exact surface $y(x)$ of a lens that bends an already convergent bundle of rays into a new bundle of rays converging toward a closer focus. 


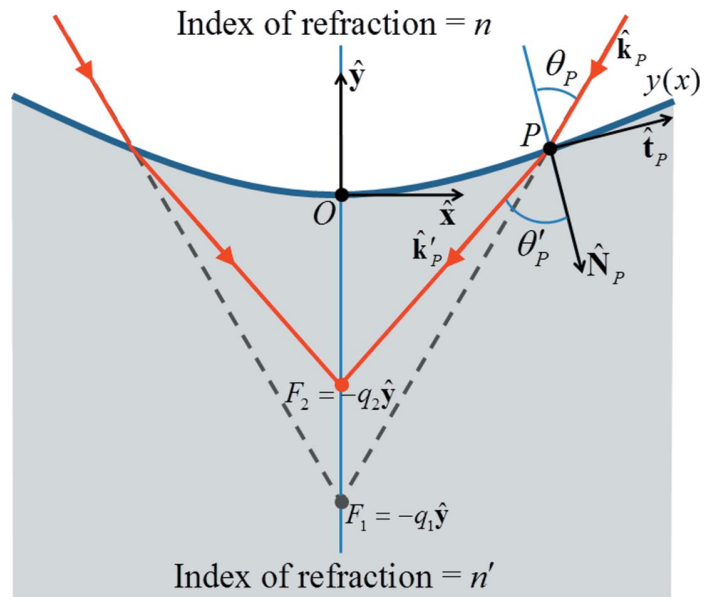

Figure 2

Schematic drawing of surface $y(x)$ (to be calculated in this paper) across which incident rays in a medium of refractive index $n$ converging to a focus $F_{1}$ are refracted into rays in a medium of refractive index $n^{\prime}$ converging to a new closer focus $F_{2} . q_{1}$ and $q_{2}$ are, respectively, the distances of $F_{1}$ and $F_{2}$ from the coordinate origin $O$ along the central axis, which is parallel to $\hat{\mathbf{y}} . \hat{\mathbf{x}}$ and $\hat{\mathbf{y}}$ are the coordinate unit vectors. $P$ is an arbitrary point along $y(x)$. $\hat{\mathbf{t}}_{P}$ and $\hat{\mathbf{N}}_{P}$ are, respectively, the unit tangent and the unit inward normal to $y(x)$ at $P . \hat{\mathbf{k}}_{P}$ and $\hat{\mathbf{k}}_{P}^{\prime}$ are, respectively, the unit wavevector of the incident ray and the refracted ray passing through $P$. $\theta_{P}$ is the angle of the incident ray to the inward normal at $P$, and $\theta_{P}^{\prime}$ is the angle of the refracted ray to the inward normal at $P$.

The required quantities are labelled and defined in Fig. 2. According to Snell's Law taken for the rays at an arbitrary point $P$,

$$
\frac{\sin \theta_{P}}{\sin \theta_{P}^{\prime}}=\frac{n^{\prime}}{n}
$$

Let the coordinate vector of $P$ be $[x \hat{\mathbf{x}}+y(x) \hat{\mathbf{y}}]$. Fig. 2 shows that

$$
\begin{gathered}
\hat{\mathbf{k}}_{P}=\frac{-x \hat{\mathbf{x}}-\left[q_{1}+y(x)\right] \hat{\mathbf{y}}}{\left\{x^{2}+\left[q_{1}+y(x)\right]^{2}\right\}^{1 / 2}}, \\
\hat{\mathbf{k}}_{P}^{\prime}=\frac{-x \hat{\mathbf{x}}-\left[q_{2}+y(x)\right] \hat{\mathbf{y}}}{\left\{x^{2}+\left[q_{2}+y(x)\right]^{2}\right\}^{1 / 2}}, \\
\hat{\mathbf{t}}_{P}=\frac{\hat{\mathbf{x}}+y^{\prime}(x) \hat{\mathbf{y}}}{\left\{1+\left[y^{\prime}(x)\right]^{2}\right\}^{1 / 2}} .
\end{gathered}
$$

It is also seen in Fig. 2 that

$$
\frac{\sin \theta_{P}}{\sin \theta_{P}^{\prime}}=\frac{\hat{\mathbf{k}}_{P} \cdot \hat{\mathbf{t}}_{P}}{\hat{\mathbf{k}}_{P}^{\prime} \cdot \hat{\mathbf{t}}_{P}} .
$$

Substitution of equations (1)-(4) into (5) yields the first-order ordinary differential equation

$$
\frac{x+y^{\prime}(x)\left[q_{1}+y(x)\right]}{\left\{x^{2}+\left[q_{1}+y(x)\right]^{2}\right\}^{1 / 2}}=\frac{n^{\prime}}{n}\left(\frac{x+y^{\prime}(x)\left[q_{2}+y(x)\right]}{\left\{x^{2}+\left[q_{2}+y(x)\right]^{2}\right\}^{1 / 2}}\right) .
$$

One can rearrange this to find an expression for the surface slope $y^{\prime}(x)$, which will be useful for design calculations once a solution for $y(x)$ has been obtained,

$$
\begin{aligned}
y^{\prime}(x)= & {\left[x\left(\frac{n^{\prime}}{n}\left\{x^{2}+\left[q_{1}+y(x)\right]^{2}\right\}^{1 / 2}-\left\{x^{2}+\left[q_{2}+y(x)\right]^{2}\right\}^{1 / 2}\right)\right] } \\
& /\left(\left[q_{1}+y(x)\right]\left\{x^{2}+\left[q_{2}+y(x)\right]^{2}\right\}^{1 / 2}\right. \\
& \left.-\frac{n^{\prime}}{n}\left[q_{2}+y(x)\right]\left\{x^{2}+\left[q_{1}+y(x)\right]^{2}\right\}^{1 / 2}\right) .
\end{aligned}
$$

This is a nonlinear differential equation, but nevertheless it can be solved by noticing that the numerators of each fraction of equation (6) are the derivatives of that fraction's denominator. A simple variable substitution thus presents itself,

$$
\begin{aligned}
& V_{1}(x)=x^{2}+\left[q_{1}+y(x)\right]^{2} \rightarrow \frac{1}{2} V_{1}^{\prime}(x)=x+y^{\prime}(x)\left[q_{1}+y(x)\right], \\
& V_{2}(x)=x^{2}+\left[q_{2}+y(x)\right]^{2} \rightarrow \frac{1}{2} V_{2}^{\prime}(x)=x+y^{\prime}(x)\left[q_{2}+y(x)\right] .
\end{aligned}
$$

As the initial condition, one may set $y(x=0)=0$ as shown in Fig. 2. In that case, $V_{1}(x=0)=q_{1}^{2}$ and $V_{2}(x=0)=q_{2}^{2}$. Integration of both sides of equation (6) starting from $x=0$ can then be written

$$
\frac{1}{2} \int_{0}^{x} \frac{V_{1}^{\prime}(s)}{\left[V_{1}(s)\right]^{1 / 2}} \mathrm{~d} s=\frac{1}{2} \frac{n^{\prime}}{n} \int_{0}^{x} \frac{V_{2}^{\prime}(s)}{\left[V_{2}(s)\right]^{1 / 2}} \mathrm{~d} s
$$

where $s$ is a dummy variable. Now, $V_{1}^{\prime}(s) \mathrm{d} s=\mathrm{d} V_{1}$ and $V_{2}^{\prime}(s) \mathrm{d} s=\mathrm{d} V_{2}$, allowing equation (10) to be rewritten in the very simple form

$$
\frac{1}{2} \int_{q_{1}^{2}}^{x^{2}+\left[q_{1}+y(x)\right]^{2}} \frac{d V_{1}}{\sqrt{V_{1}}}=\frac{1}{2} \frac{n^{\prime}}{n} \int_{q_{2}^{2}}^{x^{2}+\left[q_{2}+y(x)\right]^{2}} \frac{d V_{2}}{\sqrt{V_{2}}} .
$$

The integrals on both sides of equation (11) are elementary and yield the result

$$
\left\{x^{2}+\left[q_{1}+y(x)\right]^{2}\right\}^{1 / 2}-q_{1}=\frac{n^{\prime}}{n}\left\{x^{2}+\left[q_{2}+y(x)\right]^{2}\right\}^{1 / 2}-\frac{n^{\prime}}{n} q_{2} .
$$

Equation (12) describes a Cartesian oval with the two foci $F_{1}$ and $F_{2}$ shown in Fig. 2. Note that the distances of any point on $y(x)$ from $F_{1}$ and $F_{2}$ are $r_{1}=\left\{x^{2}+\left[q_{1}+y(x)\right]^{2}\right\}^{1 / 2}$ and $r_{2}=$ $\left\{x^{2}+\left[q_{2}+y(x)\right]^{2}\right\}^{1 / 2}$, respectively. Equation (12) can then be written in the standard form for a Cartesian oval (Weisstein, 2016),

$$
\begin{aligned}
& r_{1}-\frac{n^{\prime}}{n} r_{2}=q_{1}-\frac{n^{\prime}}{n} q_{2} \quad \text { if } \quad\left(q_{1}-\frac{n^{\prime}}{n} q_{2}\right)>0, \\
& \frac{n^{\prime}}{n} r_{2}-r_{1}=\frac{n^{\prime}}{n} q_{2}-q_{1} \quad \text { if } \quad\left(q_{1}-\frac{n^{\prime}}{n} q_{2}\right)<0 \text {. }
\end{aligned}
$$


The case $\left[q_{1}-\left(n^{\prime} / n\right) q_{2}\right]=0$ may be of some interest, and is physically achievable for the problem of this article $\left(q_{1}>q_{2}\right)$ if $n^{\prime}>n$. In this case, equation (12) can be squared and rearranged into

$$
x^{2}+\left\{y(x)+\left[\frac{q_{1}-\left(n^{\prime} / n\right)^{2} q_{2}}{1-\left(n^{\prime} / n\right)^{2}}\right]\right\}^{2}=\left[\frac{q_{1}-\left(n^{\prime} / n\right)^{2} q_{2}}{1-\left(n^{\prime} / n\right)^{2}}\right]^{2},
$$

and $q_{1}-\left(n^{\prime} / n\right)^{2} q_{2}=-\left(n^{\prime} / n\right)\left[\left(n^{\prime} / n\right)-1\right] q_{2}<0$. This is a circle of radius $\rho$ centred at $(0,-\rho)$, where

$$
\rho=\frac{\left[q_{1}-\left(n^{\prime} / n\right)^{2} q_{2}\right]}{\left[1-\left(n^{\prime} / n\right)^{2}\right]}=\left[\frac{\left(n^{\prime} / n\right)}{1+\left(n^{\prime} / n\right)}\right] q_{2}=\frac{q_{1}}{1+\left(n^{\prime} / n\right)} .
$$

\subsection{Closed-form solutions of ideal lens surface}

2.2.1. Derivation of algebraic equation. By adding $q_{1}$ to both sides of equation (12) and then squaring the equation, one obtains

$$
\begin{aligned}
x^{2}+\left[q_{1}+y(x)\right]^{2} & = \\
& \left(\frac{n^{\prime}}{n}\right)^{2}\left\{x^{2}+\left[q_{2}+y(x)\right]^{2}\right\} \\
& +2\left(\frac{n^{\prime}}{n}\right)\left[q_{1}-\left(\frac{n^{\prime}}{n}\right) q_{2}\right]\left\{x^{2}+\left[q_{2}+y(x)\right]^{2}\right\}^{1 / 2} \\
+ & {\left[q_{1}-\left(\frac{n^{\prime}}{n}\right) q_{2}\right]^{2} }
\end{aligned}
$$

Rearranging this equation to put the radical alone on one side, then squaring it again, yields

$$
\begin{aligned}
& {\left[q_{1}+y(x)\right]^{4}+\left(\frac{n^{\prime}}{n}\right)^{4}\left[q_{2}+y(x)\right]^{4}} \\
& -2\left(\frac{n^{\prime}}{n}\right)^{2}\left[q_{1}+y(x)\right]^{2}\left[q_{2}+y(x)\right]^{2} \\
& +2\left\{\left[1-\left(\frac{n^{\prime}}{n}\right)^{2}\right] x^{2}-\left[q_{1}-\frac{n^{\prime}}{n} q_{2}\right]^{2}\right\}\left[q_{1}+y(x)\right]^{2} \\
& -2\left(\frac{n^{\prime}}{n}\right)^{2}\left\{\left[1-\left(\frac{n^{\prime}}{n}\right)^{2}\right] x^{2}+\left[q_{1}-\frac{n^{\prime}}{n} q_{2}\right]^{2}\right\}\left[q_{2}+y(x)\right]^{2} \\
& +\left\{\left[1-\left(\frac{n^{\prime}}{n}\right)^{2}\right]^{2} x^{4}-2\left[1+\left(\frac{n^{\prime}}{n}\right)^{2}\right]\left[q_{1}-\frac{n^{\prime}}{n} q_{2}\right]^{2} x^{2}\right. \\
& \left.+\left[q_{1}-\frac{n^{\prime}}{n} q_{2}\right]^{4}\right\}=0 .
\end{aligned}
$$

Equation (17) is a quartic polynomial equation in both $x$ and $y$. It can be written as a quadratic equation in $x^{2}$, since no odd powers of $x$ appear in it, and by using the quadratic formula a closed-form expression of $x(y)$ can be calculated. However, the inversion of this function to obtain $y(x)$ is difficult, and $y(x)$ would be far more useful for design calculations of the lens surface. It was thus decided to solve equation (17) for $y(x)$ explicitly. Writing equation (17) in powers of $y$ yields

$$
\begin{aligned}
& {\left[1-\left(\frac{n^{\prime}}{n}\right)^{2}\right]^{2} y^{4}+4\left[1-\left(\frac{n^{\prime}}{n}\right)^{2}\right]\left[q_{1}-\left(\frac{n^{\prime}}{n}\right)^{2} q_{2}\right] y^{3}} \\
& +\left\{2\left[1-\left(\frac{n^{\prime}}{n}\right)^{2}\right]^{2} x^{2}+4\left[1-\left(\frac{n^{\prime}}{n}\right)^{2}\right]\left[q_{1}^{2}-\left(\frac{n^{\prime}}{n}\right)^{2} q_{2}^{2}\right]\right. \\
& \left.+4\left(\frac{n^{\prime}}{n}\right)\left[1-\left(\frac{n^{\prime}}{n}\right)\right]^{2} q_{1} q_{2}\right\} y^{2} \\
& +\left\{4\left[1-\left(\frac{n^{\prime}}{n}\right)^{2}\right]\left[q_{1}-\left(\frac{n^{\prime}}{n}\right)^{2} q_{2}\right] x^{2}\right. \\
& \left.+8\left(\frac{n^{\prime}}{n}\right)\left[1-\left(\frac{n^{\prime}}{n}\right)\right] q_{1} q_{2}\left[q_{1}-\left(\frac{n^{\prime}}{n}\right) q_{2}\right]\right\} y \\
& +\left(\left[1-\left(\frac{n^{\prime}}{n}\right)^{2}\right]^{2} x^{4}+4\left(\frac{n^{\prime}}{n}\right)\left\{-\left(\frac{n^{\prime}}{n}\right)\left(q_{1}^{2}+q_{2}^{2}\right)\right.\right. \\
& \left.\left.+\left[1+\left(\frac{n^{\prime}}{n}\right)^{2}\right] q_{1} q_{2}\right\} x^{2}\right)=0 .
\end{aligned}
$$

The calculation of $y(x)$ therefore amounts to finding the roots of the quartic polynomial equation (18) for any $x$. As a quartic polynomial equation with real coefficients, equation (18) is guaranteed to have four solutions, of which

- all four may be real, or

- two may be real, while the other two are complex and conjugates of each other, or

- all four may be complex, forming two pairs of complex conjugates.

No more than one of these roots can satisfy the original equation of the Cartesian oval, equation (12). To be physically significant, that root must be real. If equation (18) produces no real root that satisfies equation (12) when calculated at some particular $x$, the ideal lens surface does not exist at that $x$. This raises the possibility that the ideal lens surface may be bounded; that is, it has a maximum achievable aperture.

2.2.2. Calculation of roots of quartic polynomial equation. Analytical procedures for calculating the roots of cubic and quartic equations were worked out in the 16th century. Nonetheless, explicit solutions of such equations are published so rarely that a detailed description of the method will be useful for the reader. Note that the procedure for quartic equations includes the determination of one root of a cubic equation. Many standard mathematical texts explain the solution of cubic and quartic equations; Weisstein (2016) has been followed closely here.

The first step in solving a general quartic equation $a y^{4}+b y^{3}+c y^{2}+d y+e=0$ is the application of a coordinate transformation to a new variable $\bar{y}$ given by

$$
\bar{y}=y+\frac{b}{4 a},
$$


and the division of both sides of the general equation by $a$ such that one obtains a 'depressed quartic', that is, a quartic with no cubic term, in $\bar{y}$. This has the form $\bar{y}^{4}+p \bar{y}^{2}+q \bar{y}+r=0$, where

$$
\begin{gathered}
p=\frac{1}{a}\left(\frac{-3 b^{2}}{8 a}+c\right), \\
q=\frac{1}{a}\left(\frac{b^{3}}{8 a^{2}}-\frac{b c}{2 a}+d\right), \\
r=\frac{1}{a}\left(-\frac{3 b^{4}}{256 a^{3}}+\frac{b^{2} c}{16 a^{2}}-\frac{b d}{4 a}+e\right) .
\end{gathered}
$$

By substituting the coefficients of equation (18) into equations (19) and (20), one obtains the coordinate transformation,

$$
y=\bar{y}-\frac{q_{1}-\left(n^{\prime} / n\right)^{2} q_{2}}{1-\left(n^{\prime} / n\right)^{2}}
$$

and the depressed equation in $\bar{y}$, which has the following coefficients,

$$
\begin{aligned}
p= & {\left[1-\left(\frac{n^{\prime}}{n}\right)^{2}\right]^{-2} P\left(x^{2}\right) } \\
= & {\left[1-\left(\frac{n^{\prime}}{n}\right)^{2}\right]^{-2}\left\{2\left[1-\left(\frac{n^{\prime}}{n}\right)^{2}\right]^{2} x^{2}-2\left[1+2\left(\frac{n^{\prime}}{n}\right)^{2}\right] q_{1}^{2}\right.} \\
& -2\left(\frac{n^{\prime}}{n}\right)^{2}\left[2+\left(\frac{n^{\prime}}{n}\right)^{2}\right] q_{2}^{2} \\
& \left.+4\left(\frac{n^{\prime}}{n}\right)\left[1+\left(\frac{n^{\prime}}{n}\right)+\left(\frac{n^{\prime}}{n}\right)^{2}\right] q_{1} q_{2}\right\}
\end{aligned}
$$

$$
\begin{aligned}
q= & {\left[1-\left(\frac{n^{\prime}}{n}\right)^{2}\right]^{-3} Q } \\
= & {\left[1-\left(\frac{n^{\prime}}{n}\right)^{2}\right]^{-3}\left\{8\left(\frac{n^{\prime}}{n}\right)^{2} q_{1}^{3}-8\left(\frac{n^{\prime}}{n}\right)^{4} q_{2}^{3}\right.} \\
& -8\left(\frac{n^{\prime}}{n}\right)^{2}\left[1+2\left(\frac{n^{\prime}}{n}\right)\right] q_{1}^{2} q_{2} \\
& \left.+8\left(\frac{n^{\prime}}{n}\right)^{3}\left[2+\left(\frac{n^{\prime}}{n}\right)\right] q_{1} q_{2}^{2}\right\}
\end{aligned}
$$

$$
\begin{aligned}
& r=\left[1-\left(\frac{n^{\prime}}{n}\right)^{2}\right]^{-4} R\left(x^{2}\right) \\
& =\left[1-\left(\frac{n^{\prime}}{n}\right)^{2}\right]^{-4}\left\{\left[1-\left(\frac{n^{\prime}}{n}\right)^{2}\right]^{4} x^{4}\right. \\
& -2\left[1-\left(\frac{n^{\prime}}{n}\right)^{2}\right]^{2}\left[1+2\left(\frac{n^{\prime}}{n}\right)^{2}\right] q_{1}^{2} x^{2} \\
& -2\left(\frac{n^{\prime}}{n}\right)^{2}\left[1-\left(\frac{n^{\prime}}{n}\right)^{2}\right]^{2}\left[2+\left(\frac{n^{\prime}}{n}\right)^{2}\right] q_{2}^{2} x^{2} \\
& +4\left(\frac{n^{\prime}}{n}\right)\left[1-\left(\frac{n^{\prime}}{n}\right)^{2}\right]^{2}\left[1+\left(\frac{n^{\prime}}{n}\right)+\left(\frac{n^{\prime}}{n}\right)^{2}\right] q_{1} q_{2} x^{2} \\
& +\left[1-4\left(\frac{n^{\prime}}{n}\right)^{2}\right] q_{1}^{4}-4\left(\frac{n^{\prime}}{n}\right)\left[1-\left(\frac{n^{\prime}}{n}\right)-3\left(\frac{n^{\prime}}{n}\right)^{2}\right] q_{1}^{3} q_{2} \\
& +2\left(\frac{n^{\prime}}{n}\right)^{2}\left[2-4\left(\frac{n^{\prime}}{n}\right)-5\left(\frac{n^{\prime}}{n}\right)^{2}-4\left(\frac{n^{\prime}}{n}\right)^{3}+2\left(\frac{n^{\prime}}{n}\right)^{4}\right] q_{1}^{2} q_{2}^{2} \\
& +4\left(\frac{n^{\prime}}{n}\right)^{5}\left[3+\left(\frac{n^{\prime}}{n}\right)-\left(\frac{n^{\prime}}{n}\right)^{2}\right] q_{1} q_{2}^{3} \\
& \left.+\left(\frac{n^{\prime}}{n}\right)^{6}\left[-4+\left(\frac{n^{\prime}}{n}\right)^{2}\right] q_{2}^{4}\right\} \text {. }
\end{aligned}
$$

The strategy now is to add to both sides of the depressed equation a quantity $\left(\bar{y}^{2} u+u^{2} / 4\right)$, where $u$ is a real quantity that will be determined shortly. Knowing that $\left(\bar{y}^{4}+\bar{y}^{2} u+u^{2} / 4\right)=\left(\bar{y}^{2}+u / 2\right)^{2}$, one obtains from the depressed equation the following,

$$
\left(\bar{y}^{2}+\frac{1}{2} u\right)^{2}=(u-p) \bar{y}^{2}-q \bar{y}+\left(\frac{1}{4} u^{2}-r\right) .
$$

The left side of equation (25) is thus a perfect square. Notice that the right side of equation (25) is a quadratic equation. Therefore it too will be a perfect square if $u$ can be chosen to make its two roots equal; that is, if its discriminant $D$ equals zero,

$$
\begin{aligned}
D & =q^{2}-4(u-p)\left(\frac{1}{4} u^{2}-r\right) \\
& =u^{3}-p u^{2}-4 r u+\left(4 p r-q^{2}\right)=0 .
\end{aligned}
$$

Equation (26) is known as the 'resolvent cubic'. As a cubic equation with real coefficients, it is guaranteed to have three roots, of which either one or all will be real. The analytical solution of a general cubic equation $u^{3}+f u^{2}+g u+h=0$ begins with the calculation of two quantities $A$ and $B$, of which the general formula is shown on the left, and the value for the resolvent cubic is shown on the right,

$$
\begin{aligned}
& A=\frac{3 g-f^{2}}{9}=-\frac{4}{3} r-\frac{1}{9} p^{2}, \\
& B=\frac{9 f g-27 h-2 f^{3}}{54}=-\frac{4}{3} p r+\frac{1}{2} q^{2}+\frac{1}{27} p^{3} .
\end{aligned}
$$


The discriminant of a general cubic equation is $D_{\mathrm{c}}=A^{3}+B^{2}$.

The next step depends on the value of $D_{\text {c }}$.

(i) $D_{\mathrm{c}}>0$. One root of the cubic equation is real and the other two are complex conjugates. The real root is $-(1 / 3) f+S+T$, where $S=\left(B+\sqrt{D_{\mathrm{c}}}\right)^{1 / 3}$ and $T=$ $\left(B-\sqrt{D_{\mathrm{c}}}\right)^{1 / 3}$. For the resolvent cubic, the real root $u_{1}$ is

$$
\begin{aligned}
u_{1}\left(x^{2}\right) & =\frac{1}{3}\left[1-\left(\frac{n^{\prime}}{n}\right)^{2}\right]^{-2} P\left(x^{2}\right)+S\left(x^{2}\right)+T\left(x^{2}\right), \\
S= & {\left[1-\left(\frac{n^{\prime}}{n}\right)^{2}\right]^{-2}\left(\left[-\frac{4}{3} P\left(x^{2}\right) R\left(x^{2}\right)+\frac{1}{2} Q^{2}+\frac{1}{27} P^{3}\left(x^{2}\right)\right]\right.} \\
& +\left\{\left[-\frac{4}{3} R\left(x^{2}\right)-\frac{1}{9} P^{2}\left(x^{2}\right)\right]^{3}\right. \\
& \left.\left.+\left[-\frac{4}{3} P\left(x^{2}\right) R\left(x^{2}\right)+\frac{1}{2} Q^{2}+\frac{1}{27} P^{3}\left(x^{2}\right)\right]^{2}\right\}^{1 / 2}\right)^{1 / 3} \\
T & =\left[1-\left(\frac{n^{\prime}}{n}\right)^{2}\right]^{-2}\left(\left[-\frac{4}{3} P\left(x^{2}\right) R\left(x^{2}\right)+\frac{1}{2} Q^{2}+\frac{1}{27} P^{3}\left(x^{2}\right)\right]\right. \\
& -\left\{\left[-\frac{4}{3} R\left(x^{2}\right)-\frac{1}{9} P^{2}\left(x^{2}\right)\right]^{3}\right. \\
& \left.\left.+\left[-\frac{4}{3} P\left(x^{2}\right) R\left(x^{2}\right)+\frac{1}{2} Q^{2}+\frac{1}{27} P^{3}\left(x^{2}\right)\right]^{2}\right\}^{1 / 2}\right)^{1 / 3} .
\end{aligned}
$$

(ii) $D_{\mathrm{c}}=0$. All roots of the cubic equation are real and at least two are equal. $S$ and $T$ in equation (28) are then equal. Thus, for the resolvent cubic,

$$
\begin{aligned}
u_{1}\left(x^{2}\right)= & \frac{1}{3}\left[1-\left(\frac{n^{\prime}}{n}\right)^{2}\right]^{-2} P\left(x^{2}\right)+2 S\left(x^{2}\right) \\
S= & {\left[1-\left(\frac{n^{\prime}}{n}\right)^{2}\right]^{-2} } \\
& \times\left\{\left[-\frac{4}{3} P\left(x^{2}\right) R\left(x^{2}\right)+\frac{1}{2} Q^{2}+\frac{1}{27} P^{3}\left(x^{2}\right)\right]\right\}^{1 / 3} .
\end{aligned}
$$

(iii) $D_{\mathrm{c}}<0$. All roots of the cubic equation are real and unequal. In this case, an angle $\varphi$ is defined such that

$$
\varphi=\arccos \left(\frac{B}{\sqrt{-A^{3}}}\right)
$$

One of the real roots of the general cubic equation is then given by

$$
u_{1}=2 \sqrt{-A} \cos \left(\frac{\varphi}{3}\right)-\frac{1}{3} f
$$

which for the resolvent cubic yields

$$
\begin{aligned}
u_{1}\left(x^{2}\right)= & {\left[1-\left(\frac{n^{\prime}}{n}\right)^{2}\right]^{-2}\left\{\frac{1}{3} P\left(x^{2}\right)+2\left[\frac{4}{3} R\left(x^{2}\right)+\frac{1}{9} P^{2}\left(x^{2}\right)\right]^{1 / 2}\right.} \\
& \left.\times \cos \left[\frac{1}{3} \arccos \left(\frac{-\frac{4}{3} P\left(x^{2}\right) R\left(x^{2}\right)+\frac{1}{2} Q^{2}+\frac{1}{27} P^{3}\left(x^{2}\right)}{\left[\frac{4}{3} R\left(x^{2}\right)+\frac{1}{9} P^{2}\left(x^{2}\right)\right]^{3 / 2}}\right)\right]\right\} .
\end{aligned}
$$

Note that in all these cases one may write

$$
u_{1}\left(x^{2}\right)=\left[1-\left(\frac{n^{\prime}}{n}\right)^{2}\right]^{-2} U_{1}\left(x^{2}\right) .
$$

Substitution of $u_{1}$ into equation (25) then yields a perfect square on both sides,

$$
\left(\bar{y}^{2}+\frac{1}{2} u_{1}\right)^{2}=\left(u_{1}-p\right)\left[\bar{y}-\frac{q}{2\left(u_{1}-p\right)}\right]^{2} .
$$

If $u_{1}>p$, then equation (33) falls into two cases. In the first, one simply equates the square root of both sides,

$$
\left(\bar{y}^{2}+\frac{1}{2} u_{1}\right)=+\left(u_{1}-p\right)^{1 / 2}\left[\bar{y}-\frac{q}{2\left(u_{1}-p\right)}\right] .
$$

Equation (34) is a quadratic equation in $\bar{y}$. Its two solutions are

$$
\begin{aligned}
\bar{y}_{\mathrm{II}}= & \frac{1}{2}\left(u_{1}-p\right)^{1 / 2} \pm \frac{1}{2}\left[-\left(u_{1}+p\right)-\frac{2 q}{\left(u_{1}-p\right)^{1 / 2}}\right]^{1 / 2} \\
= & {\left[1-\left(\frac{n^{\prime}}{n}\right)^{2}\right]^{-1}\left\{\frac{1}{2}\left[U_{1}\left(x^{2}\right)-P\left(x^{2}\right)\right]^{1 / 2}\right.} \\
& \left. \pm \frac{1}{2}\left(-\left[U_{1}\left(x^{2}\right)+P\left(x^{2}\right)\right]-\frac{2 Q}{\left[U_{1}\left(x^{2}\right)-P\left(x^{2}\right)\right]^{1 / 2}}\right)^{1 / 2}\right\} .
\end{aligned}
$$

In the second case, one equates the square root of the left side of equation (33) with the negative of the square root of the right side, so that

$$
\left(\bar{y}^{2}+\frac{1}{2} u_{1}\right)=-\left(u_{1}-p\right)^{1 / 2}\left[\bar{y}-\frac{q}{2\left(u_{1}-p\right)}\right] .
$$

Equation (36), like equation (34), is a quadratic equation in $\bar{y}$. Its two solutions are

$$
\begin{aligned}
\bar{y}_{\mathrm{II} \pm}= & -\frac{1}{2}\left(u_{1}-p\right)^{1 / 2} \pm \frac{1}{2}\left[-\left(u_{1}+p\right)+\frac{2 q}{\left(u_{1}-p\right)^{1 / 2}}\right]^{1 / 2} \\
= & {\left[1-\left(\frac{n^{\prime}}{n}\right)^{2}\right]^{-1}\left(-\frac{1}{2}\left[U_{1}\left(x^{2}\right)-P\left(x^{2}\right)\right]^{1 / 2}\right.} \\
& \left. \pm \frac{1}{2}\left\{-\left[U_{1}\left(x^{2}\right)+P\left(x^{2}\right)\right]+\frac{2 Q}{\left[U_{1}\left(x^{2}\right)-P\left(x^{2}\right)\right]^{1 / 2}}\right\}^{1 / 2}\right) .
\end{aligned}
$$

$\bar{y}_{\mathrm{II}}$ and $\bar{y}_{\mathrm{II} \pm}$ are the four solutions of the depressed quartic. The explicit expressions in $P, Q$ and $U_{1}$ were calculated assuming that $1-\left(n^{\prime} / n\right)^{2}>0$; however, the same expressions are also valid if $1-\left(n^{\prime} / n\right)^{2}<0$. The only change is that the explicit expression for $\bar{y}_{\mathrm{I} \pm}$ would appear like that for $\bar{y}_{\mathrm{II} \pm}$ in equation (37), and the explicit equation for $\bar{y}_{\mathrm{II} \pm}$ would appear 
like that for $\bar{y}_{\mathrm{II}}$ in equation (35). Because this does not affect the solution, it will not be mentioned further.

The solutions of the original quartic equation (18) are easily obtained from equations (35) and (37) by using equation (21),

$$
\begin{aligned}
y_{\mathrm{II}}= & {\left[1-\left(\frac{n^{\prime}}{n}\right)^{2}\right]^{-1}\left(-\left[q_{1}-\left(\frac{n^{\prime}}{n}\right)^{2} q_{2}\right]\right.} \\
& +\frac{1}{2}\left[U_{1}\left(x^{2}\right)-P\left(x^{2}\right)\right]^{1 / 2} \\
& \left. \pm \frac{1}{2}\left\{-\left[U_{1}\left(x^{2}\right)+P\left(x^{2}\right)\right]-\frac{2 Q}{\left[U_{1}\left(x^{2}\right)-P\left(x^{2}\right)\right]^{1 / 2}}\right\}^{1 / 2}\right), \\
y_{\mathrm{II} \pm}= & {\left[1-\left(\frac{n^{\prime}}{n}\right)^{2}\right]^{-1}\left(-\left[q_{1}-\left(\frac{n^{\prime}}{n}\right)^{2} q_{2}\right]\right.} \\
& -\frac{1}{2}\left[U_{1}\left(x^{2}\right)-P\left(x^{2}\right)\right]^{1 / 2} \\
& \left. \pm \frac{1}{2}\left\{-\left[U_{1}\left(x^{2}\right)+P\left(x^{2}\right)\right]+\frac{2 Q}{\left[U_{1}\left(x^{2}\right)-P\left(x^{2}\right)\right]^{1 / 2}}\right\}^{1 / 2}\right) .
\end{aligned}
$$

In principle, any of these roots could be the one that satisfies the original equation for the Cartesian oval, equation (12). The simplest way to find this root is to evaluate equations (38) and (39) at $x=0$. The root that equals zero there is the correct one. Equation (18) will have one and only one root equal to zero at $x=0$, because then the constant $\left(y^{0}\right)$ term vanishes but the linear $\left(y^{1}\right)$ term does not.

If $u_{1}=p$, the formulas for the roots become somewhat simpler,

$$
\begin{aligned}
y_{\mathrm{I} \pm}= & {\left[1-\left(\frac{n^{\prime}}{n}\right)^{2}\right]^{-1}\left(-\left[q_{1}-\left(\frac{n^{\prime}}{n}\right)^{2} q_{2}\right]\right.} \\
& \left. \pm \frac{1}{2}\left\{-2 P\left(x^{2}\right)-2\left[P^{2}\left(x^{2}\right)-4 R\left(x^{2}\right)\right]^{1 / 2}\right\}^{1 / 2}\right), \\
y_{\mathrm{II} \pm}= & {\left[1-\left(\frac{n^{\prime}}{n}\right)^{2}\right]^{-1}\left(-\left[q_{1}-\left(\frac{n^{\prime}}{n}\right)^{2} q_{2}\right]\right.} \\
& \left. \pm \frac{1}{2}\left\{-2 P\left(x^{2}\right)+2\left[P^{2}\left(x^{2}\right)-4 R\left(x^{2}\right)\right]^{1 / 2}\right\}^{1 / 2}\right) .
\end{aligned}
$$

These roots must also be checked to determine which one fulfills equation (12) for the Cartesian oval.

If $u_{1}<p$, then equation (33) can have a real solution only if both of the squared factors are equal to zero. This would require that $\bar{y}^{2}+u_{1} / 2=0$ and $\bar{y}-q /\left[2\left(u_{1}-p\right)\right]=0$ simultaneously. If that is not possible for the values of $p, q$ and $u_{1}$ calculated above, then no real solution exists.

Fig. 3 shows a set of examples of the exact solutions in equations (38) and (39) for various values of $n^{\prime} / n$. The solutions clearly appear in two disconnected sheets, one inner and one outer. However, only one of these sheets satisfies
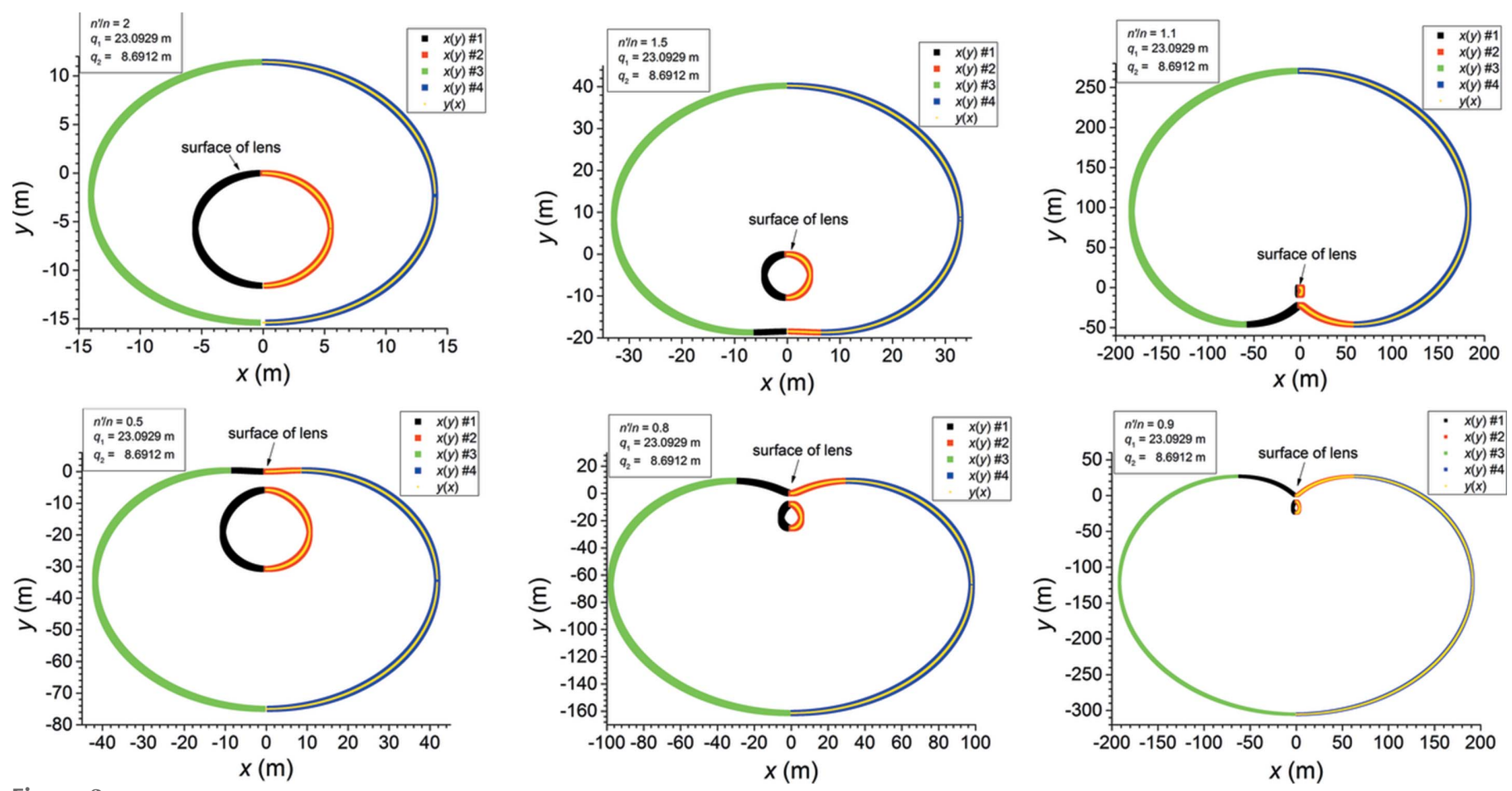

Figure 3

Examples of solutions of the Cartesian oval equation (18) at various values of $n^{\prime} / n$ for a representative set of values for $q_{1}(23.0929 \mathrm{~m})$ and $q_{2}(8.6912 \mathrm{~m})$. The solutions ' $y(x)$ ' were calculated by using the exact equations (38) and (39) for $x>0$; note that $y(-x)=y(x)$. The solutions ' $x(y)$ ' were calculated by solving equation (18) as a quadratic equation in $x^{2}$ with $y$-dependent coefficients [see equation (45)]. The top row demonstrates three cases in which $n^{\prime} / n>1$ and the bottom row demonstrates three cases in which $n^{\prime} / n<1$. In each row, $n^{\prime} / n \rightarrow 1$ from left to right. The sheet labelled 'surface of lens' is the one that fulfills the original lens equation (12). 
the original lens equation (12). If $n^{\prime} / n>1$, it is the inner sheet; if $n^{\prime} / n<1$, it is the outer sheet. It is evident that as $n^{\prime} / n \rightarrow 1$ the outer sheet becomes very much larger than the inner sheet. How large the outer sheet becomes at $x=0$ can be estimated as follows. First, define the following quantities from equations (22)-(24),

$$
\begin{gathered}
P_{0}=\lim _{n^{\prime} / n \rightarrow 1} P(x=0)=-6\left(q_{1}-q_{2}\right)^{2}, \\
Q_{0}=\lim _{n^{\prime} / n \rightarrow 1} Q=8\left(q_{1}-q_{2}\right)^{3}, \\
R_{0}=\lim _{n^{\prime} / n \rightarrow 1} R(x=0)=-3\left(q_{1}-q_{2}\right)^{4} .
\end{gathered}
$$

Substitution of these values into equation (27) yields $A=0$ and $B=0$. Thus the discriminant $D_{\mathrm{c}}$ of the resolvent cubic is zero, and from equation (29) one can define $U_{10}=$ $\lim _{n^{\prime} / n \rightarrow 1} U_{1}(x=0)=(1 / 3) P_{0}$. Because $P_{0}<0, U_{10}>P_{0}$ and equations (38) and (39) apply. Letting $\varepsilon=1-\left(n^{\prime} / n\right)^{2}$ and recalling the initial assumption $q_{1}>q_{2}$, one finds three solutions that remain bounded while the fourth solution $y_{\text {II- }}(x=0)$ diverges as $-4 \varepsilon^{-1}\left(q_{1}-q_{2}\right)$. This sensitivity of the fourth root on the exact value of $\varepsilon$ makes the quartic equation (18) ill-conditioned. Therefore the numerical evaluation of equations (38) and (39) is very sensitive to roundoff errors caused by the limited precision in cases in which $\varepsilon$ is very small, even though the equations themselves remain theoretically exact. Such cases are not only common but normal in $\mathrm{X}$-ray optics, for which $\delta=\left(n^{\prime} / n\right)-1$ generally has a magnitude on the order of $10^{-5}$ or less. An approximation that can capture the three bounded roots of equation (18) with high accuracy while ignoring the divergent root is therefore justified.

\subsection{The 'X-ray approximation' to the ideal lens surface}

Equation (18) can be rewritten as a quadratic equation in $x^{2}$ simply by rearranging terms. The quadratic formula can then be applied to determine an equation for $x^{2}$ in terms of $y$,

$$
\begin{aligned}
(\varepsilon x)^{2}= & -(\varepsilon y)^{2}-2\left[q_{1}-\left(\frac{n^{\prime}}{n}\right)^{2} q_{2}\right](\varepsilon y) \\
& +2\left(\frac{n^{\prime}}{n}\right)\left[\left(\frac{n^{\prime}}{n}\right) q_{1}-q_{2}\right]\left[q_{1}-\left(\frac{n^{\prime}}{n}\right) q_{2}\right] \\
& \times\left(1 \pm\left\{1-\frac{2\left(q_{1}-q_{2}\right)(\varepsilon y)}{\left[\left(n^{\prime} / n\right) q_{1}-q_{2}\right]^{2}}\right\}^{1 / 2}\right) .
\end{aligned}
$$

The ' \pm ' accounts for the two roots of any quadratic equation. However, if the plus sign is chosen, the resulting equation cannot be satisfied by the condition $y(x=0)=0$ as is required. Therefore only the minus sign yields a useful set of solutions for the lens surface.

Equation (45) is exact. However, the radical can be expanded by using the binomial theorem if

$$
z=\left|2\left(q_{1}-q_{2}\right)(\varepsilon y) /\left[\left(n^{\prime} / n\right) q_{1}-q_{2}\right]^{2}\right| \ll 1 .
$$

[For the X-ray case where $n^{\prime} / n \simeq 1$, this condition reduces approximately to $\left|2(\varepsilon y) /\left(q_{1}-q_{2}\right)\right| \ll 1$.] The binomial theorem yields

$$
(1+z)^{1 / 2} \simeq 1+\frac{1}{2} z-\frac{1}{8} z^{2}+\frac{1}{16} z^{3}-\frac{5}{128} z^{4}+\ldots .
$$

Hence $1-(1+z)^{1 / 2} \simeq-(1 / 2) z+(1 / 8) z^{2}-(1 / 16) z^{3}$ plus higher-order terms that will be discussed later. Note that this has no term in $z^{0}$. Substituting this into equation (45) and summing terms with the same power of $(\varepsilon y)$ on the right-hand side, one obtains the approximate equation

$$
(\varepsilon x)^{2} \simeq C_{1}(\varepsilon y)+C_{2}(\varepsilon y)^{2}+C_{3}(\varepsilon y)^{3} .
$$

For the linear term on the right-hand side, one obtains

$$
C_{1}=\frac{2\left[1-\left(n^{\prime} / n\right)-\left(n^{\prime} / n\right)^{2}+\left(n^{\prime} / n\right)^{3}\right] q_{1} q_{2}}{\left[\left(n^{\prime} / n\right) q_{1}-q_{2}\right]} .
$$

As $n^{\prime} / n \rightarrow 1$, this quantity becomes very small as the terms in the numerator almost cancel out. Equation (48) therefore becomes subject to numerical errors caused by limited precision. However, remembering that in the X-ray case $\left(n^{\prime} / n\right)=$ $1+\delta$ where $|\delta|$ is much less than 1 , one can make a power series expansion of $C_{1}$ in $\delta$. The lowest term of this power series is

$$
C_{1} \simeq \frac{4 q_{1} q_{2}}{\left(q_{1}-q_{2}\right)} \delta^{2} .
$$

For the quadratic term on the right-hand side of equation (47), one obtains

$$
\begin{aligned}
C_{2}= & \left\{\left(\frac{n^{\prime}}{n}\right)\left[1-\left(\frac{n^{\prime}}{n}\right)^{2}\right] q_{1}^{3}-2\left(\frac{n^{\prime}}{n}\right)\left[1-\left(\frac{n^{\prime}}{n}\right)\right] q_{1}^{2} q_{2}\right. \\
& \left.-2\left(\frac{n^{\prime}}{n}\right)\left[1-\left(\frac{n^{\prime}}{n}\right)\right] q_{1} q_{2}^{2}+\left[1-\left(\frac{n^{\prime}}{n}\right)^{2}\right] q_{2}^{3}\right\} \\
& /\left[\left(\frac{n^{\prime}}{n}\right) q_{1}-q_{2}\right]^{3} .
\end{aligned}
$$

Like $C_{1}, C_{2}$ also approaches zero as $\left(n^{\prime} / n\right) \rightarrow 1$. The lowest term of the power series expansion of $C_{2}$ in terms of $\delta$ is

$$
C_{2} \simeq \frac{-2\left(q_{1}+q_{2}\right)}{\left(q_{1}-q_{2}\right)} \delta .
$$

For the cubic term on the right-hand side of equation (47), one obtains

$$
C_{3}=\left(\frac{n^{\prime}}{n}\right) \frac{\left[q_{1}-\left(n^{\prime} / n\right) q_{2}\right]\left(q_{1}-q_{2}\right)^{3}}{\left[\left(n^{\prime} / n\right) q_{1}-q_{2}\right]^{5}},
$$

whose power series in terms of $\delta$ is found simply by setting $\delta=0$,

$$
C_{3} \simeq \frac{1}{\left(q_{1}-q_{2}\right)} .
$$

Finally one can calculate the lowest term in the power series expansion of $\varepsilon$,

$$
\varepsilon=1-\left(\frac{n^{\prime}}{n}\right)^{2} \simeq-2 \delta
$$


Substituting equations (49), (51), (53) and (54) into equation (47) and keeping only the lowest-order terms in $\delta$ yields

$\delta^{2} x^{2}=-\frac{2 q_{1} q_{2}}{\left(q_{1}-q_{2}\right)} \delta^{3} y-\frac{2\left(q_{1}+q_{2}\right)}{\left(q_{1}-q_{2}\right)} \delta^{3} y^{2}-\frac{2}{\left(q_{1}-q_{2}\right)} \delta^{3} y^{3}+\ldots$

It is justified to keep all terms up to cubic on the right-hand side of equation (55) because all are multiplied by the same power of $\delta$. The neglected higher-order terms $Y_{n}(n \geq 4)$ on the right-hand side of equation (55), which arise from the binomial expansion in equation (46), are given to lowest order in $\delta$ by

$$
Y_{n}=-2^{2 n-1}\left(\begin{array}{c}
1 / 2 \\
n
\end{array}\right)\left(q_{1}-q_{2}\right)^{2}\left(\frac{\delta y}{q_{1}-q_{2}}\right)^{n} .
$$

In the X-ray approximation, these terms diminish rapidly with increasing $n$. Therefore the fourth-order term is already much less than the cubic term included in the right-hand side of equation (55), and higher-order terms are smaller still. This justifies the neglect of terms beyond the cubic in equation (55).

In standard form, equation (55) is

$$
y^{3}+\left(q_{1}+q_{2}\right) y^{2}+\left(q_{1} q_{2}\right) y+\frac{\left(q_{1}-q_{2}\right)}{2 \delta} x^{2}=0 .
$$

This equation can be solved analytically. When $x=0$ the roots are trivial: $0,-q_{1}$ and $-q_{2}$. The solutions for general $x$ can be determined by the same methods used to calculate the resolvent cubic of the exact equation. The discriminant of equation (57) is $D_{\mathrm{XR}}=A_{\mathrm{XR}}^{3}+B_{\mathrm{XR}}^{2}$, where

$$
\begin{gathered}
A_{\mathrm{XR}}=-\frac{1}{9}\left(q_{1}^{2}-q_{1} q_{2}+q_{2}^{2}\right), \\
B_{\mathrm{XR}}(x)=-\frac{\left(q_{1}-q_{2}\right)}{4 \delta} x^{2}+\frac{1}{54}\left(q_{1}+q_{2}\right)\left[9 q_{1} q_{2}-2\left(q_{1}+q_{2}\right)^{2}\right] .
\end{gathered}
$$

Notice that, since $q_{1}>0$ and $q_{2}>0, A_{\mathrm{XR}}<0$ because $\left(q_{1}^{2}-q_{1} q_{2}+q_{2}^{2}\right)=\left(q_{1}-q_{2}\right)^{2}+q_{1} q_{2}>0$. From these expressions, one obtains

$$
\begin{aligned}
D_{\mathrm{XR}}(x)= & \frac{\left(q_{1}-q_{2}\right)^{2}}{16 \delta^{2}} x^{4} \\
& -\frac{1}{108 \delta}\left(q_{1}-q_{2}\right)\left(q_{1}+q_{2}\right)\left[9 q_{1} q_{2}-2\left(q_{1}+q_{2}\right)^{2}\right] x^{2} \\
& -\frac{1}{108}\left(q_{1} q_{2}\right)^{2}\left(q_{1}-q_{2}\right)^{2} .
\end{aligned}
$$

Now one needs to determine the sign of $D_{\mathrm{XR}}$ at any given $x$. Notice that $D_{\mathrm{XR}}$ depends quadratically on $x^{2}$. Therefore one can use the quadratic formula to find the values $x_{D 0}$ at which $D_{\mathrm{XR}}=0$,

$$
\begin{aligned}
x_{D 0 \pm}^{2}= & \frac{4 \delta}{q_{1}-q_{2}}\left\{\frac{1}{54}\left(q_{1}+q_{2}\right)\left[9 q_{1} q_{2}-2\left(q_{1}+q_{2}\right)^{2}\right]\right. \\
& \left. \pm \frac{1}{9^{3 / 2}}\left(q_{1}^{2}-q_{1} q_{2}+q_{2}^{2}\right)^{3 / 2}\right\} \\
= & \frac{4 \delta}{q_{1}-q_{2}}\left[B_{\mathrm{XR} 0} \pm\left(-A_{\mathrm{XR}}\right)^{3 / 2}\right],
\end{aligned}
$$

where $B_{\mathrm{XR} 0}=B_{\mathrm{XR}}(x=0)$. From this one finds that

$$
x_{D 0+}^{2} x_{D 0-}^{2}=\frac{16 \delta^{2}}{\left(q_{1}-q_{2}\right)^{2}}\left[B_{\mathrm{XR} 0}^{2}+A_{\mathrm{XR}}^{3}\right]=\frac{16 \delta^{2}}{\left(q_{1}-q_{2}\right)^{2}} D_{\mathrm{XR} 0},
$$

where $D_{\mathrm{XR} 0}=D_{\mathrm{XR}}(x=0)$. Inspection of equation (60) shows that $D_{\mathrm{XR} 0}<0$ and that therefore $x_{D 0+}^{2} x_{D 0-}^{2}<0$, which proves that $x_{D 0+}^{2}$ and $x_{D 0-}^{2}$ have opposite signs. Equation (61) shows that, if $\delta /\left(q_{1}-q_{2}\right)>0, \quad x_{D 0+}^{2}>0$ and $x_{D 0-}^{2}<0$, since $\left(-A_{\mathrm{XR}}\right)^{3 / 2}>0$. Likewise, if $\delta /\left(q_{1}-q_{2}\right)<0, x_{D 0+}^{2}<0$ and $x_{D 0-}^{2}>0$. Only the positive squared $x$ can yield real values of $x$; the negative squared $x$ is discarded. There are thus two values of $x$ at which the discriminant $D_{\mathrm{XR}}(x)=0$ :

(i) $\delta /\left(q_{1}-q_{2}\right)>0$.

$$
\begin{aligned}
x= & \pm x_{D 0}= \pm 2\left(\frac{\delta}{q_{1}-q_{2}}\right)^{1 / 2}\left[B_{\mathrm{XR} 0}+\left(-A_{\mathrm{XR}}\right)^{3 / 2}\right]^{1 / 2} \\
= & \pm 2\left(\frac{\delta}{q_{1}-q_{2}}\right)^{1 / 2}\left\{\frac{1}{54}\left(q_{1}+q_{2}\right)\left[9 q_{1} q_{2}-2\left(q_{1}+q_{2}\right)^{2}\right]\right. \\
& \left.+\frac{1}{9^{3 / 2}}\left(q_{1}^{2}-q_{1} q_{2}+q_{2}^{2}\right)^{3 / 2}\right\}^{1 / 2} .
\end{aligned}
$$

(ii) $\delta /\left(q_{1}-q_{2}\right)<0$.

$$
\begin{aligned}
x= & \pm x_{D 0}= \pm 2\left(-\frac{\delta}{q_{1}-q_{2}}\right)^{1 / 2}\left[-B_{\mathrm{XR} 0}+\left(-A_{\mathrm{XR}}\right)^{3 / 2}\right]^{1 / 2} \\
= & \pm 2\left(-\frac{\delta}{q_{1}-q_{2}}\right)^{1 / 2}\left\{-\frac{1}{54}\left(q_{1}+q_{2}\right)\left[9 q_{1} q_{2}-2\left(q_{1}+q_{2}\right)^{2}\right]\right. \\
& \left.+\frac{1}{9^{3 / 2}}\left(q_{1}^{2}-q_{1} q_{2}+q_{2}^{2}\right)^{3 / 2}\right\}^{1 / 2} .
\end{aligned}
$$

The positive coefficient of the $x^{4}$ term in equation (60) shows that $D_{\mathrm{XR}}(x)$ increases with increasing $x^{2}$. Therefore, the solutions of equation (57) are as follows:

(a) $|x|<x_{D 0}$, all values of $\delta$. Here the discriminant $D_{\mathrm{XR}}$ of equation (57) is negative. In this case the three roots of equation (57) are all real and unequal. The calculation of the roots begins with the calculation of an angle $\beta$ such that

$$
\beta_{\mathrm{XR}}(x)=\arccos \left[\frac{B_{\mathrm{XR}}(x)}{\left(-A_{\mathrm{XR}}\right)^{3 / 2}}\right] .
$$

The roots are then

$$
y_{\mathrm{XR} 1}(x)=2 \sqrt{-A_{\mathrm{XR}}} \cos \left[\frac{\beta_{\mathrm{XR}}(x)}{3}\right]-\frac{1}{3}\left(q_{1}+q_{2}\right),
$$

$$
\begin{aligned}
y_{\mathrm{XR} 2}(x)= & -\sqrt{-A_{\mathrm{XR}}}\left\{\cos \left[\frac{\beta_{\mathrm{XR}}(x)}{3}\right]+\sqrt{3} \sin \left[\frac{\beta_{\mathrm{XR}}(x)}{3}\right]\right\} \\
& -\frac{1}{3}\left(q_{1}+q_{2}\right), \\
y_{\mathrm{XR} 3}(x)= & -\sqrt{-A_{\mathrm{XR}}}\left\{\cos \left[\frac{\beta_{\mathrm{XR}}(x)}{3}\right]-\sqrt{3} \sin \left[\frac{\beta_{\mathrm{XR}}(x)}{3}\right]\right\} \\
& -\frac{1}{3}\left(q_{1}+q_{2}\right) .
\end{aligned}
$$


One can now check these roots at $x=0$, defining $\beta_{\mathrm{XR} 0}=$ $\beta_{\mathrm{XR}}(x=0)$. By using the trigonometric identity $\cos \theta=$ $4 \cos ^{3}(\theta / 3)-3 \cos (\theta / 3)$, one can show that if $(1 / 3)\left(q_{1}+q_{2}\right) /\left(2 \sqrt{-A_{\mathrm{XR}}}\right)=\cos \left(\beta_{\mathrm{XR} 0} / 3\right)$, then $\cos \beta_{\mathrm{XR} 0}=$ $B_{\mathrm{XR} 0} /\left(-A_{\mathrm{XR}}\right)^{3 / 2}$, thus satisfying equation (65). One can also use the common trigonometric identity $\sin ^{2} \theta+\cos ^{2} \theta=1$ to find that, for $q_{1}>q_{2}$ as assumed here, $\sin \left(\beta_{\mathrm{XR} 0} / 3\right)=$ $(1 / \sqrt{3})\left(q_{1}-q_{2}\right) /\left(2 \sqrt{-A_{\mathrm{XR}}}\right)$. Thus $y_{\mathrm{XR} 1}(x=0)=0$, $y_{\mathrm{XR} 2}(x=0)=-q_{1}$ and $y_{\mathrm{XR} 3}(x=0)=-q_{2}$, as expected. Note that $y_{\mathrm{XR} 1}(x)$ is the solution for the shape of the lens.

(b) $|x|=x_{D 0}, \delta>0$ (assuming $q_{1}>q_{2}$ ). The discriminant $D_{\mathrm{XR}}\left( \pm x_{D 0}\right)=0$. Therefore the roots are all real and two of them are equal. Substitution of equation (63) into equation (59) shows that in this case $B_{\mathrm{XR}}\left( \pm x_{D 0}\right)=-\left(-A_{\mathrm{XR}}\right)^{3 / 2}$. Therefore, according to equation (65), $\beta_{\mathrm{XR}}\left( \pm x_{D 0}\right)=$ $\arccos (-1)=\pi$. Using equations $(66)-(68)$, one finds the roots

$$
\begin{gathered}
y_{\mathrm{XR} 1}\left( \pm x_{D 0}\right)=\sqrt{-A_{\mathrm{XR}}}-\frac{1}{3}\left(q_{1}+q_{2}\right), \\
y_{\mathrm{XR} 2}\left( \pm x_{D 0}\right)=-2 \sqrt{-A_{\mathrm{XR}}}-\frac{1}{3}\left(q_{1}+q_{2}\right), \\
y_{\mathrm{XR} 3}\left( \pm x_{D 0}\right)=\sqrt{-A_{\mathrm{XR}}}-\frac{1}{3}\left(q_{1}+q_{2}\right) .
\end{gathered}
$$

Therefore, in this case, $y_{\mathrm{XR} 1}(x)$ and $y_{\mathrm{XR} 3}(x)$ together form the inner sheet of the Cartesian oval. Since we know that $y_{\mathrm{XR} 1}(x)$ is the desired lens surface, this is consistent with the exact quartic equation.

(c) $|x|=x_{D 0}, \delta<0$ (assuming $q_{1}>q_{2}$ ). The discriminant $D_{\mathrm{XR}}\left( \pm x_{D 0}\right)=0$. Therefore the roots are all real and two of them are equal. Substitution of equation (64) into equation (59) shows that in this case $B_{\mathrm{XR}}\left( \pm x_{D 0}\right)=+\left(-A_{\mathrm{XR}}\right)^{3 / 2}$. Therefore, according to equation (65), $\beta_{\mathrm{XR}}\left( \pm x_{D 0}\right)=$ $\arccos (+1)=0$. Using equations $(66)-(68)$, one finds the roots

$$
\begin{aligned}
& y_{\mathrm{XR} 1}\left( \pm x_{D 0}\right)=2 \sqrt{-A_{\mathrm{XR}}}-\frac{1}{3}\left(q_{1}+q_{2}\right), \\
& y_{\mathrm{XR} 2}\left( \pm x_{D 0}\right)=-\sqrt{-A_{\mathrm{XR}}}-\frac{1}{3}\left(q_{1}+q_{2}\right), \\
& y_{\mathrm{XR} 3}\left( \pm x_{D 0}\right)=-\sqrt{-A_{\mathrm{XR}}}-\frac{1}{3}\left(q_{1}+q_{2}\right) .
\end{aligned}
$$

Therefore, in this case, $y_{\mathrm{XR} 2}(x)$ and $y_{\mathrm{XR} 3}(x)$ form the inner sheet of the Cartesian oval, and $y_{\mathrm{XR} 1}(x)$ must be on the Cartesian oval's outer sheet.

(d) $|x|>x_{D 0}, \delta>0$ (assuming $q_{1}>q_{2}$ ). The discriminant $D_{\mathrm{XR}}$ is now positive [see equation (60)]. Therefore only one real root exists. (The other two are complex and hence not physically significant.) This root must join up with $y_{\mathrm{XR} 2}(x)$ in equation (70). The real root is given by the expression

$$
\begin{aligned}
y_{\mathrm{XR} 2}(x)= & \left\{B_{\mathrm{XR}}(x)+\left[D_{\mathrm{XR}}(x)\right]^{1 / 2}\right\}^{1 / 3} \\
& +\left\{B_{\mathrm{XR}}(x)-\left[D_{\mathrm{XR}}(x)\right]^{1 / 2}\right\}^{1 / 3}-\frac{1}{3}\left(q_{1}+q_{2}\right) .
\end{aligned}
$$

As $x \rightarrow \pm x_{D 0}, y_{\mathrm{XR} 2}(x) \rightarrow 2\left[B_{\mathrm{XR}}\left( \pm x_{D 0}\right)\right]^{1 / 3}-(1 / 3)\left(q_{1}+q_{2}\right)=$ $-2 \sqrt{-A_{\mathrm{XR}}}-(1 / 3)\left(q_{1}+q_{2}\right)$, which does indeed join up with equation (70) as expected. Note that this does not form part of the solution to the lens surface, but it is included here for completeness.

(e) $|x|>x_{D 0}, \delta<0$ (assuming $q_{1}>q_{2}$ ). Again, as the discriminant $D_{\mathrm{XR}}$ is positive, only one real root exists. This root must join up with $y_{\mathrm{XR} 1}(x)$ in equation (72),

$$
\begin{aligned}
y_{\mathrm{XR} 1}(x)= & \left\{B_{\mathrm{XR}}(x)+\left[D_{\mathrm{XR}}(x)\right]^{1 / 2}\right\}^{1 / 3} \\
& +\left\{B_{\mathrm{XR}}(x)-\left[D_{\mathrm{XR}}(x)\right]^{1 / 2}\right\}^{1 / 3}-\frac{1}{3}\left(q_{1}+q_{2}\right) .
\end{aligned}
$$

As $x \rightarrow \pm x_{D 0}, y_{\mathrm{XR} 1}(x) \rightarrow 2\left[B_{\mathrm{XR}}\left( \pm x_{D 0}\right)\right]^{1 / 3}-(1 / 3)\left(q_{1}+q_{2}\right)=$ $+2 \sqrt{-A_{\mathrm{XR}}}-(1 / 3)\left(q_{1}+q_{2}\right)$, which does indeed join up with equation (72) as expected. This does form part of the solution to the lens surface.

Examples of the cubic X-ray approximation of the Cartesian oval are shown in Fig. 4. The outputs displayed in these graphs were calculated using MATLAB (MathWorks, 2004) in the default double precision. At $|\delta|=10^{-4}$, the exact equation for the Cartesian oval is still well conditioned enough to deliver stable output, and the X-ray approximation already agrees well with it. It is at values of $\delta$ below this that the usefulness of the X-ray approximation becomes obvious. Attempts to use the exact formula result in inconsistent output, while the output of the X-ray approximation remains stable.

Fig. 5 displays a series of SHADOW3 ray-tracing simulations (Sanchez del Rio et al., 2011) of the lens surfaces determined by using the X-ray approximation for the six cases in Fig. 4. All of the 500000 rays originate from a two-dimensional Gaussian source of $1 \mu \mathrm{m}$ height and width. This is much smaller than a normal synchrotron electron beam source, but was chosen to keep down aberrations that appear when the source size becomes comparable with the lens aperture. The rays are randomly sampled in angle over a uniform distribution of horizontal width $1.6 \mu \mathrm{rad}$ and vertical width $1.6 \mu \mathrm{rad}$. These widths were chosen in order to just exactly cover the full aperture of the lens surface. Two optical elements are used in each simulation. The first optical element is used solely to turn the divergent rays from the source point into a convergent beam. It is a perfectly reflecting, ideally shaped ellipsoidal mirror located $23.10287 \mathrm{~m}$ from the source point. This mirror is set to a central grazing incidence angle of $3 \mathrm{mrad}$. It is shaped so that its source point coincides with the original source of the rays and its image point lies $23.10287 \mathrm{~m}$ downstream. The second optical element is the lens surface. It is located $0.010 \mathrm{~m}$ downstream from the first optical element. The image point lies $8.6912 \mathrm{~m}$ downstream. The basic shape is a plane surface of aperture $0.07425 \mathrm{~mm}$ horizontal $\times$ $0.07425 \mathrm{~mm}$ vertical. To this plane is added a spline interpolation generated by the SHADOW3 utility PRESURFACE from a $501 \times 501$ mesh of points calculated by $M A T L A B$ from the X-ray approximation of this section. The index of refraction is taken as constant in the medium upstream from the lens surface and in the medium downstream from the lens surface. Absorption is neglected in both media. The displayed plots are all taken at the final focal point. In all six simulations, the 

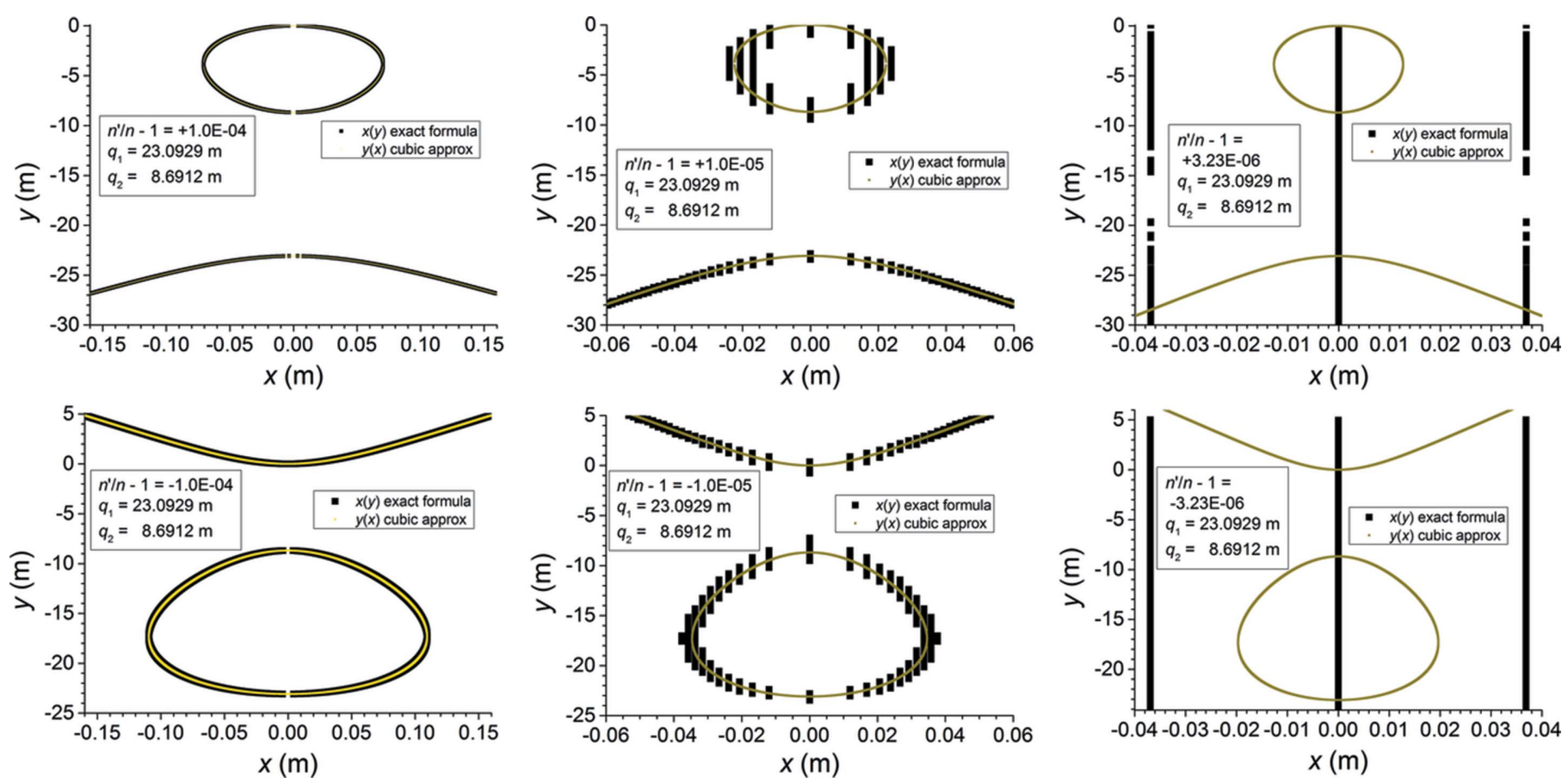

Figure 4

Examples of solutions of the cubic X-ray approximation [equation (57)] at various values of $\delta$ for a representative set of values for $q_{1}(23.0929 \mathrm{~m})$ and $q_{2}(8.6912 \mathrm{~m})$. The solutions of the cubic approximation are labelled $y(x)$. The solutions ' $x(y)$ ' were calculated by solving equation (18) as a quadratic equation in $x^{2}$ with $y$-dependent coefficients [see equation (45)]. The top row demonstrates three cases in which $\delta>0$ and the bottom row demonstrates three cases in which $\delta<0$. In each row, $\delta \rightarrow 0$ from left to right. Note the loss of numerical precision in the solutions of the exact formula as $|\delta|$ decreases, even while the cubic approximation remains numerically stable.

distribution of rays in the image fits well to Gaussians of FWHM very close to $0.886 \mu \mathrm{m}$, the geometrical demagnified source size, in both height and width. A calculation of the spot size using the SHADOW3 utility RAY_PROP on 17 frames over a range within $\pm 0.8 \mathrm{~m}$ from the image point at $8.6912 \mathrm{~m}$ showed that this point was indeed, as required, the point at which the rays converged (see Fig. 6). It is therefore demonstrated that the X-ray approximation can indeed generate lens surfaces that focus convergent beam.

\subsection{The paraxial approximation to a conic section}

If the incident rays deviate from the central line $x=0$ by only a small amount, the calculations of the ideal lens surface and of the lens surface in the X-ray approximation both show that the value $y(x)$ of the lens surface will also be small. In this case, one can assume that the cubic term in equation (47) is much smaller than the quadratic term, thus leading to the condition

$$
\left|\frac{C_{3}}{C_{2}}(\varepsilon y)\right| \ll 1,
$$

for which the cubic term in equation (47) can be neglected. [Recall that $\varepsilon=1-\left(n^{\prime} / n\right)^{2}$ and that $C_{2}$ and $C_{3}$ are defined in equations (50) and (52), respectively.] In the X-ray approximation, equation (77) reduces to the simple condition

$$
\frac{|y|}{q_{1}+q_{2}} \ll 1 .
$$

If equation (77) (for the general case) or equation (78) (for the $\mathrm{X}$-ray approximation) is fulfilled, then the paraxial approximation is valid. Equation (47) then reduces to

$$
(\varepsilon x)^{2} \simeq C_{1}(\varepsilon y)+C_{2}(\varepsilon y)^{2},
$$

and equation (57) for the X-ray approximation reduces to

$$
\left(q_{1}+q_{2}\right) y^{2}+\left(q_{1} q_{2}\right) y+\frac{\left(q_{1}-q_{2}\right)}{2 \delta} x^{2}=0 .
$$

The solutions $y(x)$ of these equations are conic sections. The type of conic section depends on the sign of the quadratic term $y^{2}$. Beginning with general values of $n^{\prime} / n$, one can complete the square of equation (79) for two cases:

(i) $C_{2}<0$. The paraxial approximation to the ideal lens surface is the ellipse

$$
\frac{x^{2}}{\left[C_{1} /\left(2 \sqrt{\left|C_{2}\right|} \varepsilon\right)\right]^{2}}+\frac{\left[y-C_{1} /\left(2\left|C_{2}\right| \varepsilon\right)\right]^{2}}{\left[C_{1} /\left(2\left|C_{2}\right| \varepsilon\right)\right]^{2}}=1 .
$$

(ii) $C_{2}>0$. The paraxial approximation to the ideal lens surface is the hyperbola

$$
\frac{\left[y+C_{1} /\left(2 C_{2} \varepsilon\right)\right]^{2}}{\left[C_{1} /\left(2 C_{2} \varepsilon\right)\right]^{2}}-\frac{x^{2}}{\left[C_{1} /\left(2 \sqrt{C_{2}} \varepsilon\right)\right]^{2}}=1 .
$$



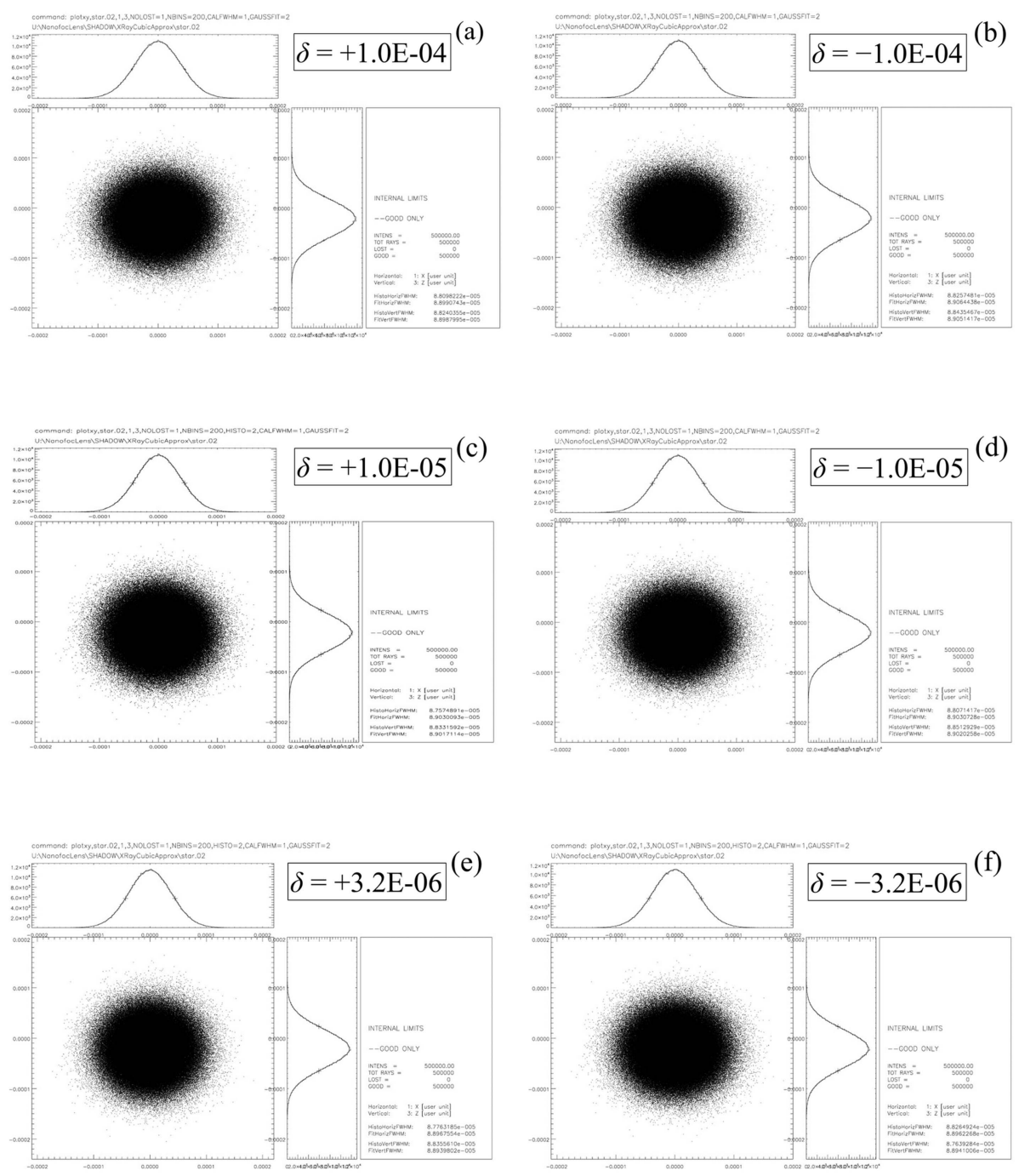

Figure 5

Six ray-tracing simulations performed by SHADOW3 on the cases displayed in Fig. 4, except for a slight modification in $(e)$ and $(f)$ to accommodate the limited precision given by SHADOW3 to the index of refraction. The values of $\delta=n^{\prime} / n-1$ are shown on each diagram. In all simulations, $q_{1}$ is $23.0929 \mathrm{~m}$ and $q_{2}$ is $8.6912 \mathrm{~m}$ as in Fig. 4 . All of the rays originate from a Gaussian source of $1 \mu \mathrm{m}$ r.m.s. height and width. They are caused by an ideal primary focusing element to converge to the initial focus $x=0, y=-q_{1}$. The refractive surface given by the X-ray approximation in each case is calculated on a $501 \times 501$ mesh of points over an aperture $0.07425 \mathrm{~mm} \times 0.07425 \mathrm{~mm}$. The SHADOW3 utility PRESURFACE is used to determine a spline function over this mesh. The resulting spline is then added to a refractive plane surface, which thus causes the rays from the primary focusing element to converge onto the final focal point $x=0, y=-q_{2}$. Units of distance are centimetres. See text for further details.

In the X-ray approximation, one can complete the square of equation (80) for two cases:

(i) $\delta>0$. The paraxial approximation to the lens surface is the ellipse

$$
\begin{gathered}
x^{2} /\left\{(\delta / 2)^{1 / 2} \frac{q_{1} q_{2}}{\left[\left(q_{1}+q_{2}\right)\left(q_{1}-q_{2}\right)\right]^{1 / 2}}\right\}^{2} \\
+\frac{\left[y+(1 / 2) q_{1} q_{2} /\left(q_{1}+q_{2}\right)\right]^{2}}{\left[(1 / 2) q_{1} q_{2} /\left(q_{1}+q_{2}\right)\right]^{2}}=1 .
\end{gathered}
$$

(ii) $\delta<0$. The paraxial approximation to the lens surface is the hyperbola

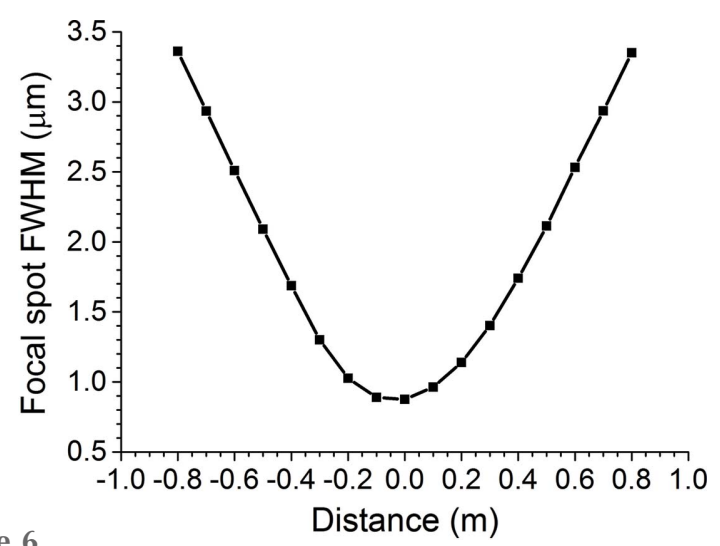

Figure 6

Depth of focus calculation of lens surface calculated by the X-ray approximation. A distance of zero denotes the image point at $8.6912 \mathrm{~m}$ downstream from the lens surface. 


$$
\begin{aligned}
& \frac{\left[y+(1 / 2) q_{1} q_{2} /\left(q_{1}+q_{2}\right)\right]^{2}}{\left[(1 / 2) q_{1} q_{2} /\left(q_{1}+q_{2}\right)\right]^{2}} \\
& -x^{2} /\left\{(|\delta| / 2)^{1 / 2} \frac{q_{1} q_{2}}{\left[\left(q_{1}+q_{2}\right)\left(q_{1}-q_{2}\right)\right]^{1 / 2}}\right\}^{2}=1 .
\end{aligned}
$$

In the limit $q_{1} \rightarrow+\infty$ and $|\delta| \ll 1$, equations (83) and (84) approach the conic sections calculated by Sanchez del Rio \& Alianelli (2012).

An even stricter paraxial approximation is obtained if, in addition to the condition given in equations (77) or (78), one demands that the quadratic $y^{2}$ term in equations (79) or (80) be much smaller than the linear $y$ term. For general $n^{\prime} / n$, this imposes the additional requirement

$$
\left|\frac{C_{2}}{C_{1}}(\varepsilon y)\right| \ll 1,
$$

which in the X-ray approximation becomes

$$
\frac{q_{1}+q_{2}}{q_{1} q_{2}}|y| \ll 1
$$

[One can see that equation (86) is in fact more stringent than equation (78) by showing that $1 /\left(q_{1}+q_{2}\right)<$ $\left(q_{1}+q_{2}\right) / q_{1} q_{2}$ for positive $q_{1}$ and $q_{2}$.] If the conditions of equations (85) or (86) are fulfilled, the lens surface may be approximated as a parabola. For general $n^{\prime} / n$, the lens surface is then approximately

$$
\frac{\varepsilon}{C_{1}} x^{2}=y,
$$

and in the X-ray approximation the lens surface is approximately

$$
-\frac{1}{2 \delta} \frac{\left(q_{1}-q_{2}\right)}{q_{1} q_{2}} x^{2}=-\frac{x^{2}}{2 \delta F}=y,
$$

where $F$ is the geometrical focal length. Double differentiation of equation (88) yields the well known relationship between the radius $R$ and the focal length $F$ of a single lens surface in the $\mathrm{X}$-ray approximation, $F=R / \delta$.

\section{Testing the paraxial approximation}

Figs. 7 and 8 demonstrate how, in the paraxial approximation, the best conic section (ellipse for $\delta>0$, hyperbola for $\delta<0$ ) and the best parabola deviate from the X-ray approximation to the ideal Cartesian oval for $\delta=$ $\pm 3.23 \times 10^{-6}$, the value for diamond at $15 \mathrm{keV}$. Surfaces 2, 24 and 48 were selected from Table 1 to demonstrate

Figure 7 slope $d y / d x$ diverges. that the conic section approximations fail at decreasing apertures as the curvature of the surface increases. As mentioned by previous authors, the parabola deviates from the X-ray approximation at much smaller apertures than does the best ellipse or hyperbola. Each plot's horizontal axis is scaled to make visible the aperture at which even the best ellipse or hyperbola begins to deviate from the X-ray approximation. Thus, for surface 2, which has an aperture of $74.25 \mu \mathrm{m}$, one would expect the parabolic approximation to be sufficient because it matches the X-ray approximation well out to $|x|<$ $2500 \mu \mathrm{m}$. For surface 24, which has an aperture of $59.52 \mu \mathrm{m}$, the parabolic approximation could still be sufficient, but, as the parabola only matches the X-ray approximation out to $|x|<200 \mu \mathrm{m}$, one might prefer to give this surface an elliptical or hyperbolic shape. For surface 48 , which has an aperture of $46.760 \mu \mathrm{m}$, even the elliptical/hyperbolic approximation begins to fail at the edges; therefore, this surface must follow the ideal curve. As a result, surface 48 was chosen for the
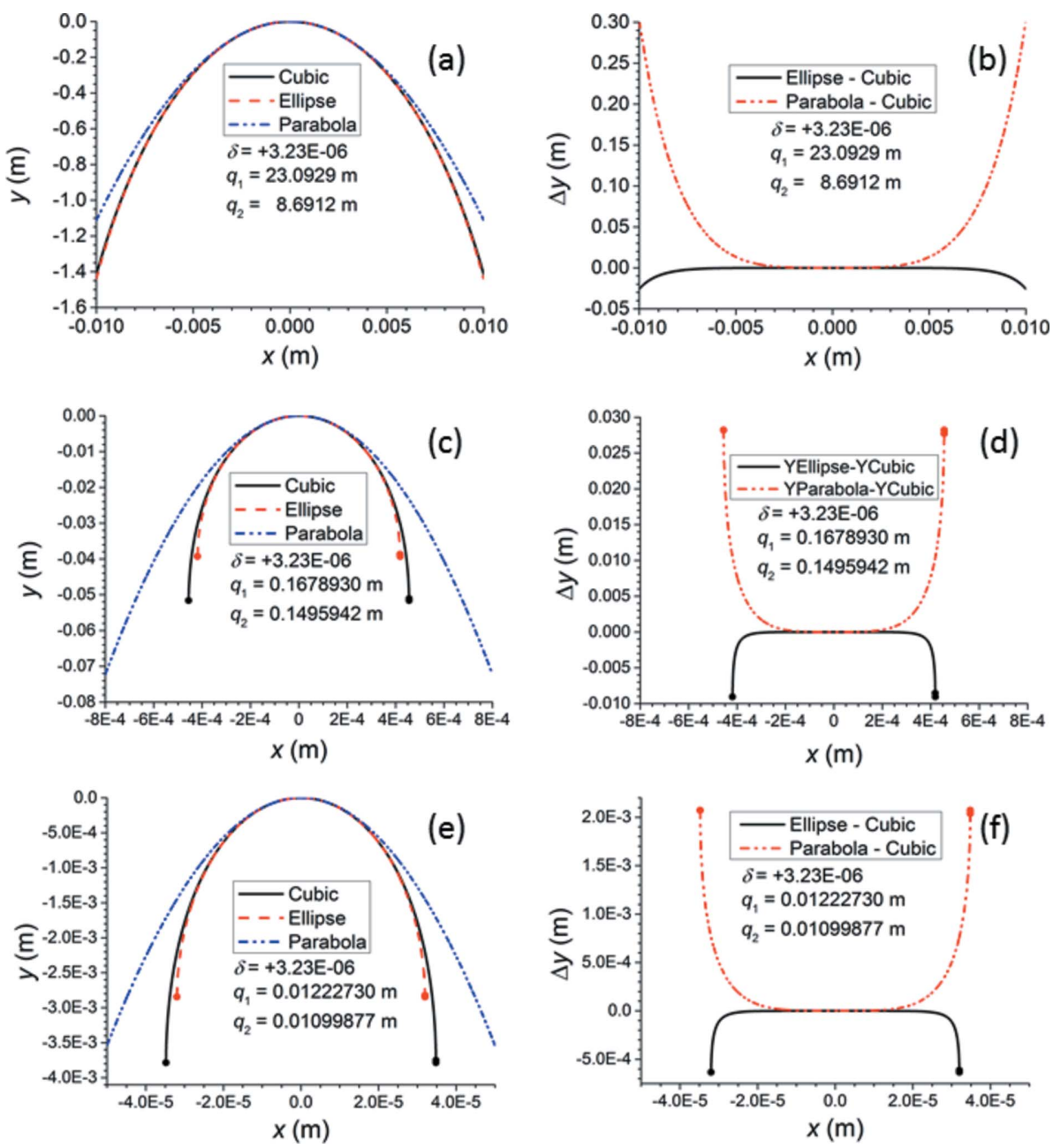

In all plots, $\delta=+3.23 \times 10^{-6}$. The label 'Cubic' means that the X-ray approximation of the ideal Cartesian oval was used to calculate the curve. Refer to Table 1 for the list of surfaces. $(a, c, e)$ Comparison of cubic curve to paraxial ellipse and parabola of surfaces 2, 24 and 48, respectively. $(b, d, f)$ Deviation of paraxial ellipse and parabola from cubic curve of surfaces 2,24 and 48 , respectively. Solid circles at the ends of a curve indicate that the curve terminates there because the 

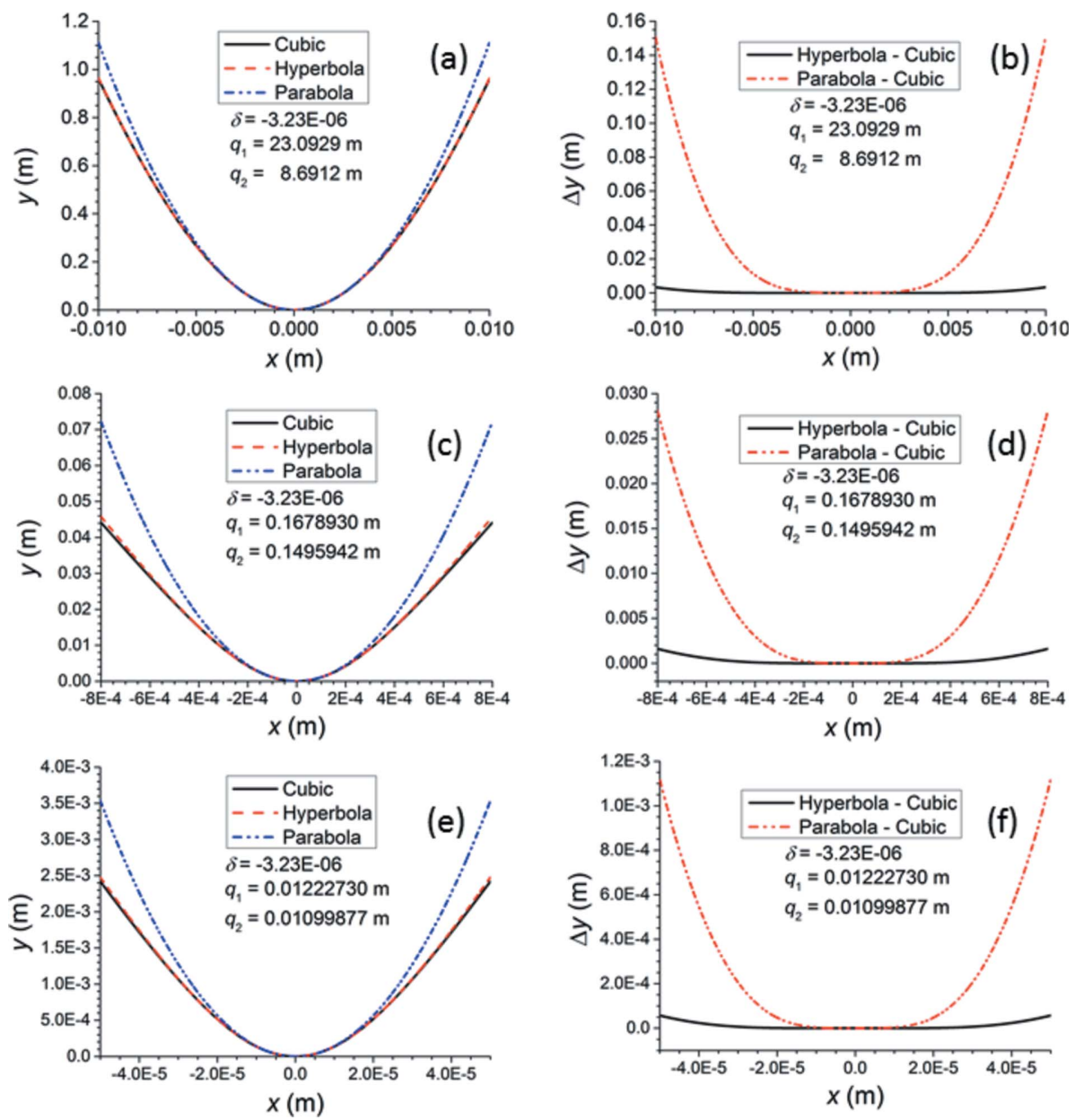

Figure 8

In all plots, $\delta=-3.23 \times 10^{-6}$. The label 'Cubic' means that the X-ray approximation of the ideal Cartesian oval was used to calculate the curve. Refer to Table 1 for the list of surfaces. $(a, c, e)$ Comparison of cubic curve to paraxial hyperbola and parabola of surfaces 2, 24 and 48, respectively. $(b, d, f)$ Deviation of paraxial hyperbola and parabola from cubic curve of surfaces 2, 24 and 48, respectively.

SHADOW ray traces of Fig. 9. To emphasize the improvement offered by the X-ray approximation over the ellipse/hyperbola, the aperture of surface 48 was slightly widened to $63.600 \mu \mathrm{m}$, at which Figs. 7 and 8 show that the ellipse or hyperbola fails severely at the edges. A value $\delta= \pm 3.2 \times 10^{-6}$ was chosen because of the limited precision given to the index of refraction input in SHADOW. In each simulation, 500000 rays were randomly selected from a Gaussian source of root mean square width $0.1 \mu \mathrm{m}$ (FWHM $0.23548 \mu \mathrm{m}$ ) and uniform angular distribution. Although the chosen size of the source is much smaller than the electron beam sizes of real synchrotron storage rings, it is applied here to approximate a true point source, eliminating aberrations that would appear in the focal spot if the source size were comparable with the lens surface's aperture. Two optical elements were created. The first was a purely theoretical spherical mirror designed to reflect all rays from the source at normal incidence. This element exists only to produce the necessary convergent beam for the second element, which is the lens surface itself. The second element is situated $12.227 \mathrm{~mm}$ upstream from the focus of the spherical mirror. It is simulated with a plane figure to which a spline file generated by the SHADOW utility PRESURFACE is added. MATLAB was used to calculate a cylinder for one-dimensional focusing with 501 points over a width of $46.760 \mu \mathrm{m}$ in the non-focusing direction and 681 points over a width of $63.600 \mu \mathrm{m}$ in the focusing direction. The rays in the calculated profiles were sorted into 250 bins according to their position. Figs. $9(a)$ and $9(c)$ show the beam profiles generated at the nominal focus $10.999 \mathrm{~mm}$ downstream from surface 48 , comparing them with the original source. The profiles generated by the ellipse or hyperbola are slightly but noticeably lower at the peak and have slightly larger tails than those generated by the $\mathrm{X}$-ray approximation to the ideal curve. The profiles generated by the parabola show a loss of about $50 \%$ of the peak intensity and correspondingly severe tails. Depth of focus plots showing the variation of the beam size versus the distance along the beam direction from the nominal focus were generated by the SHADOW3 utility $R A Y \_P R O P$ and are displayed in Figs. $9(b)$ and $9(d)$. The X-ray approximation yields a lens surface that minimizes the beam width at the nominal focus, as required. The FWHM of the beam profile at this minimum is $0.210 \mu \mathrm{m}$, which matches the geometrically demagnified source size. The ellipse/hyperbola shifts the minimum of the beam width closer to the lens surface by $10-20 \mu \mathrm{m}$, and this minimum is still not quite as small as that achieved by the $\mathrm{X}$-ray approximation. The parabola shifts the minimum of the beam width by about $60-70 \mu \mathrm{m}$ from the nominal focus, and this minimum is considerably larger than that achieved by either the X-ray approximation or the ellipse/hyperbola. These results again demonstrate that the $\mathrm{X}$-ray approximation can generate lens surfaces that focus convergent beam better than the approximate conic sections can do.

\section{Diffraction broadening}

SHADOW was used to calculate the effective aperture of the CRL in Table 1. 500000 rays of $15 \mathrm{keV}$ energy were created from a point source. They were uniformly distributed in angle so that the entire geometrical aperture of the first lens surface $(0.075 \mathrm{~mm} \times 0.075 \mathrm{~mm})$ was illuminated. Each lens surface was assumed to be a parabolic cylinder, focusing in the vertical direction only. All of the lens surfaces except the first were taken to be unbounded so that their limited geometrical 

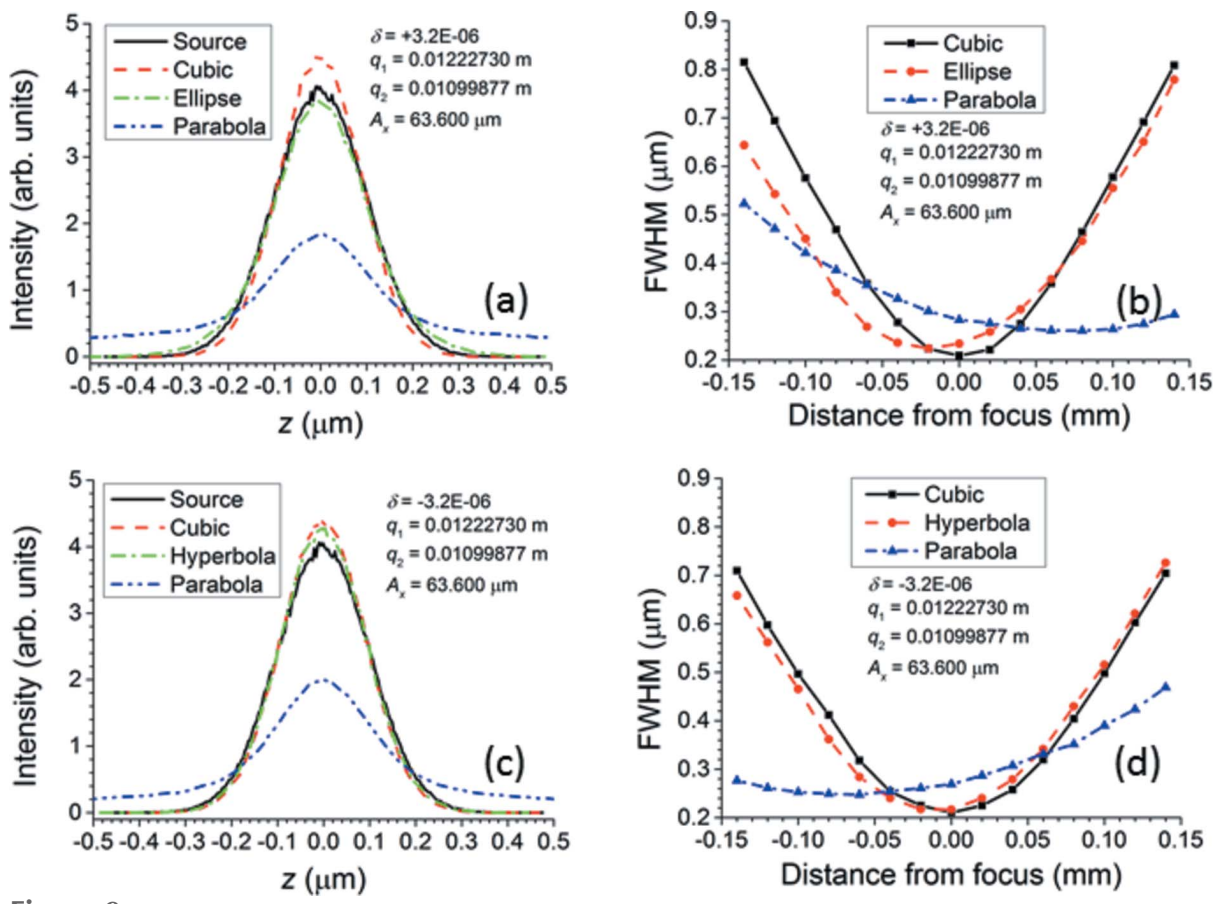

Figure 9

SHADOW ray-tracing calculations using surface 48 of Table 1 over a geometrical aperture $A_{x}$ of $63.600 \mu \mathrm{m}$. The source is a Gaussian with a root mean square width of $0.1 \mu \mathrm{m}$. $(a, c)$ Profiles of source and of focal spots produced by the cubic curve, the paraxial conic section (ellipse/hyperbola) and the parabola for $\delta=+3.2 \times 10^{-6}$ and $\delta=-3.2 \times 10^{-6}$, respectively. $(b, d)$ FWHM of focal spots produced by the cubic curve, the paraxial conic section (ellipse/hyperbola) and the parabola for $\delta=+3.2 \times 10^{-6}$ and $\delta=-3.2 \times 10^{-6}$, respectively, as a function of distance along the beam from the nominal focus. See text for details.

apertures would not cut off any of the rays propagating inside the CRL. The lens material is diamond, which for $15 \mathrm{keV}$ $\mathrm{X}$-rays has an index of refraction differing from 1 in its real part by $-3.23 \times 10^{-6}$. The linear absorption coefficient $\mu$ is $0.282629 \mathrm{~mm}^{-1}$. The calculated intensity distribution on the last surface (number 48) and the calculated angular distribution of the intensity converging onto the final focus are displayed in Figs. $10(a)$ and $10(b)$, respectively. The effective aperture is the FWHM of the plot in Fig. 10(a), $26.47 \mu \mathrm{m}$. The corresponding numerical aperture is half the FWHM of the angular plot in Fig. 10(b), 1.204 mrad. The diffraction broadening therefore amounts to $0.75 \lambda /(2 \mathrm{NA})=25.74 \mathrm{~nm}$, which is much less than the focal spot widths in Fig. 9. Moreover, within the FWHM effective aperture in Fig. 10(a), a parabola is still a sufficiently good approximation to the ideal shape of the final lens surface, as shown in Figs. 7(e) and $7(f)$.

\section{Conclusions}

The immediate goal of this paper was to prove that an analytical solution, namely a Cartesian oval, exists for a lens surface that is to refocus an incident beam converging to a point into a new beam converging to a point closer to the lens surface. This result serves the long-term goal of designing aberration-free aspherical CRLs that will in future produce X-ray beam spots of $50 \mathrm{~nm}$ width and, further on, even $10 \mathrm{~nm}$ width. Numerical difficulties that arose in the analytical calculation of the Cartesian oval when the change in refractive index across the lens surface is small, as is usual for X-ray optics, were overcome by a cubic approximation that was numerically stable. The focusing performance of lens surfaces following the cubic 'X-ray' approximation was compared with that of lens surfaces shaped either as ellipses or hyperbolas, or as parabolas, as previous authors have suggested. Elliptical or hyperbolic lens surfaces yield stronger peaks and lower tails at the focus than do parabolic lens surfaces, but surfaces that follow the cubic X-ray approximation provide better focal profiles than either. Examples taken from a proposed adiabatically focusing lens, in which the radius of curvature and the aperture of the lens surfaces both decrease along the beam direction, indicate that the advantages of the X-ray approximation over conic sections are most apparent in the final, most strongly curved, lenses.

\section{Acknowledgements}

L. A. would like to thank M. Sanchez del Rio (ESRF) for the many illuminating discussions on optics and on numerical methods in SHADOW.
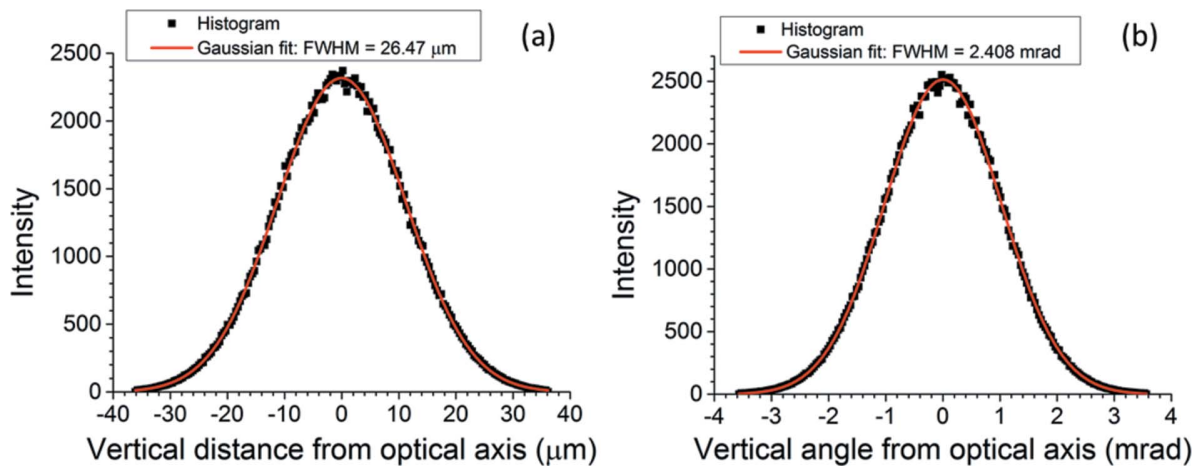

Figure 10

(a) Vertical intensity distribution calculated by $S H A D O W$ ray trace on final lens surface (number 48) of the CRL in Table 1. (b) Vertical angular distribution of intensity calculated by SHADOW ray trace at the final focus of the CRL of Table 1. Each histogram sorts the 500000 rays of the simulation into 200 bins. See text for details. 


\section{References}

Alianelli, L., Laundy, D., Alcock, S., Sutter, J. \& Sawhney, K. (2016). Synchrotron Radiat. News, 29(4), 3-9.

Alianelli, L., Sánchez del Rio, M., Fox, O. J. L. \& Korwin-Mikke, K. (2015). Opt. Lett. 40, 5586-5589.

Alianelli, L., Sawhney, K. J. S., Barrett, R., Pape, I., Malik, A. \& Wilson, M. C. (2011). Opt. Express, 19, 11120-11127.

Aristov, V., Grigoriev, M., Kuznetsov, S., Shabelnikov, L., Yunkin, V., Weitkamp, T., Rau, C., Snigireva, I., Snigirev, A., Hoffmann, M. \& Voges, E. (2000). Appl. Phys. Lett. 77, 4058-4060.

Evans-Lutterodt, K., Ablett, J. M., Stein, A., Kao, C.-C., Tennant, D. M., Klemens, F., Taylor, A., Jacobsen, C., Gammel, P. L., Huggins, H., Ustin, S., Bogart, G. \& Ocola, L. (2003). Opt. Express, 11, 919-926.

Evans-Lutterodt, K., Stein, A., Ablett, J. M., Bozovic, N., Taylor, A. \& Tennant, D. M. (2007). Phys. Rev. Lett. 99, 134801.

Fox, O. J. L., Alianelli, L., Malik, A. M., Pape, I., May, P. W. \& Sawhney, K. J. S. (2014). Opt. Express, 22, 7657-7668.
Kohn, V. G. (2017). J. Synchrotron Rad. 24, 609-614.

Lengeler, B., Schroer, C., Tümmler, J., Benner, B., Richwin, M., Snigirev, A., Snigireva, I. \& Drakopoulos, M. (1999). J. Synchrotron Rad. 6, 1153-1167.

Lengeler, B., Tümmler, J., Snigirev, A., Snigireva, I. \& Raven, C. (1998). J. Appl. Phys. 84, 5855-5861.

MathWorks (2004). MATLAB Version 7.0.1.24704 (R14) Service Pack 1. MathWorks, Inc., Natick, Massachusetts, USA.

Nöhammer, B., Hoszowska, J., Freund, A. K. \& David, C. (2003). J. Synchrotron Rad. 10, 168-171.

Sanchez del Rio, M. \& Alianelli, L. (2012). J. Synchrotron Rad. 19, 366-374.

Sanchez del Rio, M., Canestrari, N., Jiang, F. \& Cerrina, F. (2011). J. Synchrotron Rad. 18, 708-716.

Schroer, C. G. \& Lengeler, B. (2005). Phys. Rev. Lett. 94, 054802.

Snigirev, A., Kohn, V., Snigireva, I. \& Lengeler, B. (1996). Nature (London), 384, 49-51.

Suzuki, Y. (2004). Jpn. J. Appl. Phys. 43, 7311-7314.

Weisstein, E. W. (2016). MathWorld - A Wolfram Web Resource, http://mathworld.wolfram.com. 